\title{
Endocytosis of FcaR is clathrin and dynamin dependent, but its cytoplasmic domain is not required
}

\author{
Min Peng ${ }^{1}$, Na Yin ${ }^{1}$, Wei Zhang ${ }^{1}$ \\ ${ }^{I}$ Institute of Basic Medical Sciences, Chinese Academy of Medical Sciences, School of Basic Medicine, Peking Union Medical Col- \\ lege, Beijing 100005, China
}

FcaR, the Fc receptor for IgA, is essential for IgA-mediated immune responses. Previous studies have shown that IgA and IgA immune complexes can be rapidly endocytosed by FcaR. However, the underlying mechanism remains unclear. Here, we investigated the endocytic pathway of $F c \alpha R$ in monocytic cell line, U937, that naturally express FcaR and in transfected Chinese hamster ovary (CHO), COS-7 and Hela cells. By using selective chemical inhibitors of different endocytic pathways, overexpression of dominant-negative mutants of Eps15 and knockdown of clathrin heavy chain (CHC) via RNA interference, we demonstrated that endocytosis of FcaR was through a clathrin-mediated pathway. The endocytosed $F c \alpha R$ went into Rab5- and Rab11-positive endosomes. However, endocytosis of FcaR could not be blocked by a dominant-negative mutant of Rab5. We also demonstrated that endocytosis of FcoR was dynamin-dependent by overexpressing a dominant-negative mutant of dynamin. The potential endocytic motif for FcaR was also examined. Unexpectedly, we found that the entire cytoplasmic domain of FcaR was not required for the endocytic process of FcaR. We conclude that endocytosis of FcaR is clathrin- and dynamin-dependent, but is not regulated by Rab5, and the endocytic motif is not located in the cytoplasmic domain of FcaR.

Keywords: IgA, Fc receptor, endocytosis, clathrin, dynamin

Cell Research (2010) 20:223-237. doi: 10.1038/cr.2009.120; published online 27 October 2009

\section{Introduction}

In humans, IgA is the most abundant antibody in mucosal surfaces and the second most abundant antibody in blood [1, 2]. IgA plays important roles in both mucosal and systemic immunity [3, 4]. Fc $\alpha \mathrm{R}$ (CD89), the $\mathrm{Fc}$ receptor for IgA, plays crucial roles in IgA-mediated immune responses by coupling the innate and adaptive immune responses in effector cell activation. Binding of antigen-complexed IgA to Fc $\alpha \mathrm{R}$ initiates a variety of responses, and endocytosis is one of them $[5,6]$.

It has been known for a long time that $\operatorname{IgA}$ and $\operatorname{IgA}$ complexed immune complex can be internalized by FcaR [7]. However, the underlying mechanism remains unknown. Since FcaR is devoid of recognized signaling

Correspondence: Wei Zhang

Tel: +86-10-65296417; Fax: +86-10-65284074

E-mail:wzhang@pumc.edu.cn

Received 25 March 2009; revised 28 June 2009; accepted 19 August 2009; published online 27 October 2009 motifs in its cytoplasmic domain, it is believed that the immune responses elicited by antigen-complexed IgA are dependent on its functional association with the FcR $\gamma$ chain, which contains ITAM (immunoreceptor tyrosinebased activation motif), a signaling motif shared by other immune receptors, such as Fc $\gamma R I$, Fc $\gamma$ RIII, BCR, TCR, etc [8-10]. Although the $\gamma$-chain contains a well-characterized endocytic motif (YXXL), which plays important roles in endocytosis and antigen presentation [11], several studies have confirmed that $\gamma$-chain is not required for Fc $\alpha \mathrm{R}$-mediated endocytosis of IgA and IgA immune complexes $[9,12]$. Instead, $\gamma$-chain is involved in the intracellular trafficking of $\mathrm{Fc} \alpha \mathrm{R}$ after endocytosis $[12,13]$. Launay et al. [12] observed that internalized IgA was recycled back to the cell surface when Fc $\alpha \mathrm{R}$ was not associated with the $\gamma$-chain, whereas internalized $\operatorname{IgA}$ was delivered to lysosome for degradation after endocytosis when Fc $\alpha \mathrm{R}$ is associated with the $\gamma$-chain. Therefore, endocytosis of IgA by $\gamma$-chain-unassociated Fc $\alpha$ R may play important roles in maintaining serum IgA concentration by protecting it from degradation.

Endocytosis of membrane receptors can occur through 
various pathways. Clathrin-dependent and clathrinindependent pathways represent the two major routes for internalization of cell surface receptors [14-16]. Clathrinmediated endocytosis, the most thoroughly studied endocytic pathway, is characterized by formation of clathrincoated pits at the plasma membrane. Forming the coated pits requires several components including clathrin, AP-2, AP-180, Eps15, etc. Clathrin-dependent endocytosis requires the GTPase dynamin [17-19], which participates in the budding of clathrin-coated vesicles that are then destined for endosomal compartments. On the other hand, clathrin-independent endocytosis is less well understood until recently [20]. Several endocytic pathways belong to this category, including caveolae-mediated endocytosis and lipid raft-mediated endocytosis, as well as pathways that are both clathrin- and caveolae/lipid raft independent [20]. For example, a recent study has shown that the high-affinity IgE receptor (FceRI) is endocytosed by an AP-2/clathrin-independent mechanism, which appears to be lipid raft-mediated and regulated by dynamin [21].

Although there are different endocytic pathways for receptors to enter cells, the internalization, intracellular trafficking and membrane fusion of endocytic vesicles are generally regulated by a subfamily of Ras-like small GTPases (Rab GTPases) [22, 23]. Approximately 40 members of Rab GTPases have been identified, and each is believed to be specifically associated with a particular organelle or pathway. Among them, Rab5 is localized in clathrin-coated vesicles and early endosomes, regulating internalization and early endosome fusion [24, 25]; Rab11 is localized in recycling endosomes, regulating the endocytic recycling [26-28]; Rab7 and Rab9 are localized in late endosomes, regulating the transport from early to late endosomes and late endsomes to the transGolgi, respectively [29-32]. It is known that endocytosis and intracellular trafficking of many $\mathrm{G}$ protein-coupled receptors are controlled by Rab GTPases [33].

In the present study, we demonstrate that $\mathrm{Fc} \alpha \mathrm{R}$ is endocytosed through the clathrin-dependent pathway and the internalized receptor sequentially goes into Rab5and Rab11-positive endosomes. Endocytosis of FcaR requires dynamin, but is not regulated by Rab5. Furthermore, we find that the cytoplasmic domain of FcaR is dispensable for its endocytosis.

\section{Results}

\section{Endocytosis of FcaR in U937 cells}

The U937 cell is a human cell line with monocytic characteristics and naturally expresses Fc $\alpha$ R. Previous study shows that FcaR undergoes rapid endocytosis in
U937 cells [12]. Therefore, U937 cells were used to investigate the endocytic pathway(s) of FcaR. Several chemical inhibitors were used to selectively block clathrin- or caveolae/lipid raft-mediated endocytosis, which are two endocytic pathways commonly used by many membrane receptors. As shown in Figures 1A and 1B, blocking clathrin-mediated endocytosis by hypertonic sucrose $(0.4 \mathrm{M}), \mathrm{MDC}(100 \mu \mathrm{M})$ and $\mathrm{K}^{+}$depletion [34-36] dramatically inhibited $\mathrm{Fc} \alpha \mathrm{R}$ internalization. In comparison, FcaR endocytosis was hardly inhibited when cells were treated with Filipin III $(5 \mu \mathrm{g} / \mathrm{ml})$ or nystatin $(50 \mu \mathrm{g} /$ $\mathrm{ml}$ ), which is able to inhibit caveolae/lipid raft-mediated endocytosis [37-39].

Clathrin-dependent pathway does not but some clathrin-independent pathways require tyrosine kinase activity for ligand internalization [40-42]. So, we examined whether tyrosine kinase activity was required for FcaR endocytosis by using tyrosine kinase inhibitors, genistein and herbimycin A. As shown in Figures $1 \mathrm{~A}$ and $1 \mathrm{~B}$, treatment of cells by genistein $(100 \mu \mathrm{g} / \mathrm{ml})$ or herbimycin A $(1 \mu \mathrm{M})$ had no influence on Fc $\alpha \mathrm{R}$ endocytosis, suggesting that tyrosine kinase activity was not required for $F c \alpha R$ endocytosis and further indicating that the endocytosis was mediated by a clathrin-dependent pathway.

\section{Endocytosis of FcaR in transfected $\mathrm{CHO}$ cells}

To study mechanisms of $F c \alpha R$ endocytosis, a stable transfectant of FcaR in $\mathrm{CHO}$ cells was established. Flow cytometry analysis showed that transfected $\mathrm{CHO}$ cells expressed FcaR on cell surface (Figure 2A) and were able to bind IgA (Figure 2B). The molecular mass of expressed $\mathrm{Fc} \alpha \mathrm{R}$ was between 55 and $75 \mathrm{kDa}$ (Figure 2C), which was similar to the naturally expressed receptor.

To investigate whether the endocytic pathway of Fc $\alpha R$ in $\mathrm{CHO}$ cells is the same as that in U937 cells, which naturally express $\mathrm{F} c \alpha \mathrm{R}$, we performed chemical inhibitor assays in $\mathrm{CHO}$ cells stably expressing FcaR. As shown in Figures 2D and 2E, both confocal microscopy and flow cytometry analysis showed that hypertonic sucrose $(0.4 \mathrm{M})$, MDC $(100 \mu \mathrm{M})$ and $\mathrm{K}^{+}$depletion dramatically inhibited Fc $\alpha \mathrm{R}$ endocytosis, whereas Filipin III $(5 \mu \mathrm{g} / \mathrm{ml})$ or nystatin $(50 \mu \mathrm{g} / \mathrm{ml})$ was unable to do so. Tyrosine kinase inhibitors, genistein $(100 \mu \mathrm{g} / \mathrm{ml})$ and herbimycin A $(1 \mu \mathrm{M})$, were also unable to inhibit Fc $\alpha \mathrm{R}$ endocytosis in $\mathrm{CHO}$ cells. As a control, $\mathrm{CHO}$ cells that were not transfected with Fc $\alpha \mathrm{R}$ did not bind or internalize IgA or FITC-conjugated MIP8a, demonstrating that endocytosis of IgA or FITC-conjugated MIP8a in transfected CHO cells was mediated by FcaR, but not other unidentified receptors (data not shown). Taken together, these results suggested that endocytosis of $\mathrm{Fc} \alpha \mathrm{R}$ in $\mathrm{CHO}$ cells followed the same pathway as that in U937 cells. 
A
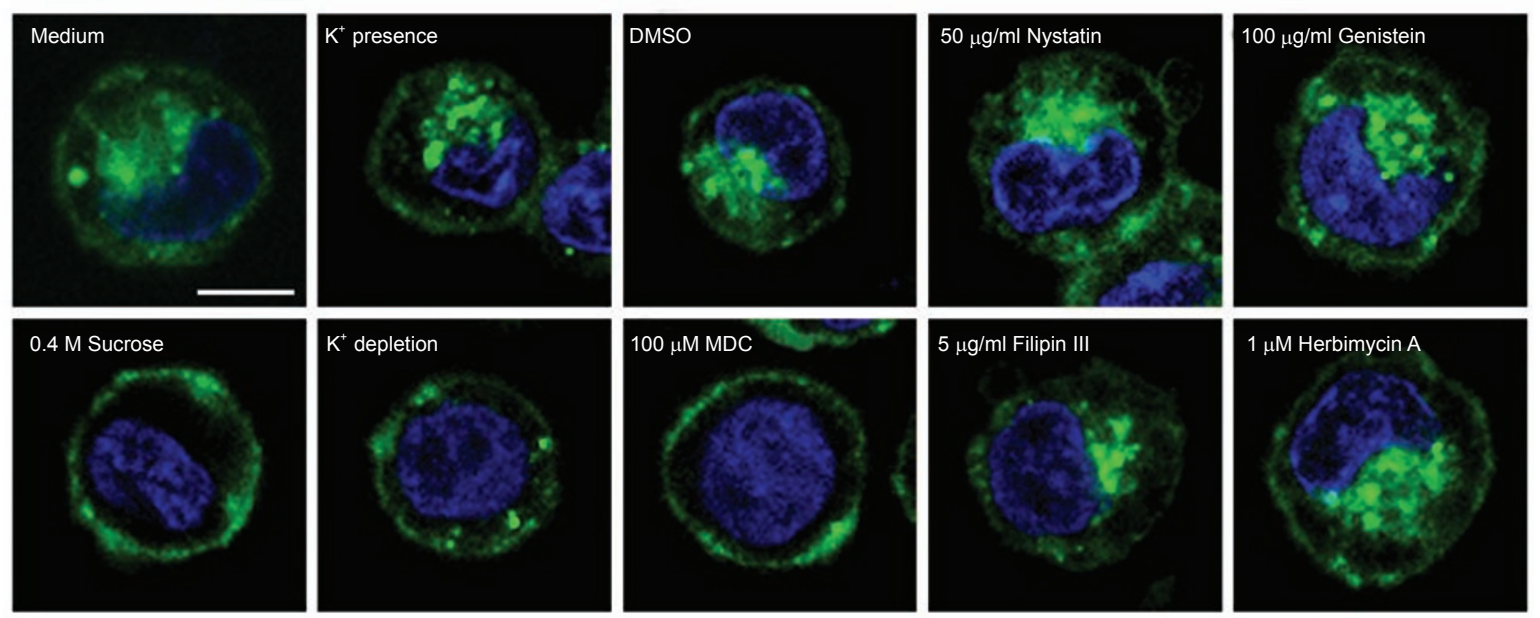

B

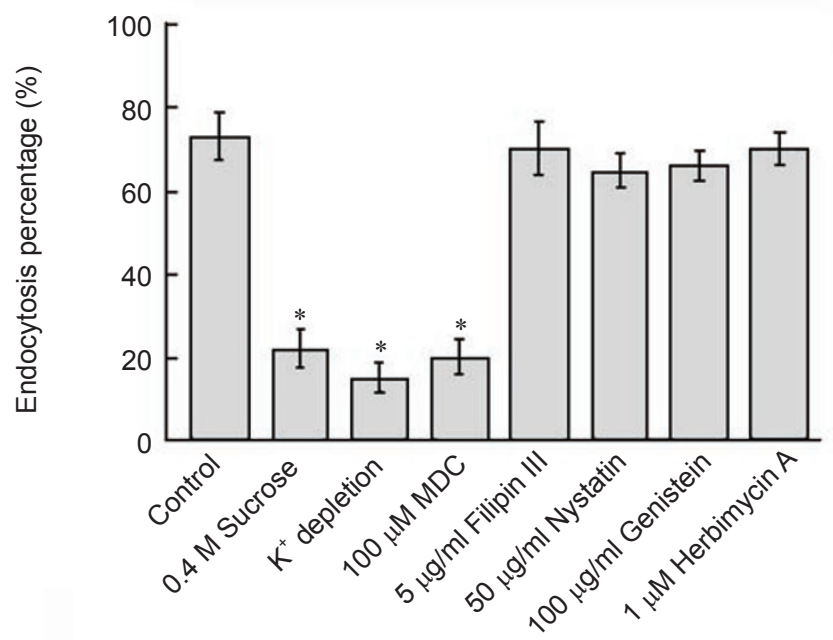

Figure 1 Endocytosis of FcaR in U937 cells. (A) U937 cells that grew on poly-lysine coated coverslip were treated with PMA $\left(10^{-7} \mathrm{M}\right)$ for $24 \mathrm{~h}$. Then cells were treated with hypertonic sucrose $(0.4 \mathrm{M})$, MDC $(100 \mu \mathrm{M})$, Filipin III $(5 \mu \mathrm{g} / \mathrm{ml})$, nystatin $(50$ $\mu \mathrm{g} / \mathrm{ml})$, genistein $(100 \mu \mathrm{g} / \mathrm{ml})$ or herbimycin $\mathrm{A}(1 \mu \mathrm{M})$ for $60 \mathrm{~min}$ at $37^{\circ} \mathrm{C}$, or subjected to $\mathrm{K}^{+}$depletion as described in Materials and Methods. Cells were then incubated with FITC-labeled MIP8a-F $\left(a b^{\prime}\right)_{2}$ in medium containing inhibitors for another 60 min at $4{ }^{\circ} \mathrm{C}$, washed and transferred quickly to pre-warmed $\left(37^{\circ} \mathrm{C}\right)$ medium containing inhibitors and incubated for 30 min to allow endocytosis. Then, cells were fixed and nuclei were stained by Hoechst 33258 (blue). Endocytosis was examined by confocal laser-scanning microscope. Bar represents $7.5 \mu \mathrm{m}$. Data are representative of three independent experiments. (B) Quantitative analysis of the effect of chemical inhibitors on FcaR endocytosis by flow cytometry, see Materials and Methods. $* P<0.01$.

\section{Dominant-negative mutants of Eps 15 inhibit FcaR inter- nalization}

Eps15 is a protein that binds directly to the plasma membrane adaptor AP-2 and is required for clathrinmediated endocytosis. Overexpression of dominantnegative mutants of Eps15 can selectively block clathrinmediated endocytosis [43]. So, we overexpressed dominant-negative mutants of Eps 15 to further examine whether Fc $\alpha$ R was internalized via clathrin-mediated endocytosis. EGFP-tagged Eps15-D3 $\Delta 2$ (wild-type (WT) control, which has no influence on clathrin-mediated endocytosis) and two EGFP-tagged dominant-negative mutants, Eps15-DIII and Eps15-EH29, were transiently transfected into $\mathrm{CHO}$ cells stably expressing FcaR. The inhibitory effect of Eps 15 mutants on clathrin-mediated endocytosis was examined by uptake of Texas Redconjugated transferrin (Texas Red-Tfn). As shown in Figures $3 \mathrm{~A}$ and $3 \mathrm{~B}$, uptake of Texas Red-Tfn was inhibited by up to $40 \%-60 \%$ when cells were transfected with EGFP-tagged Eps15-EH29 and Eps15-DIII. The WT control EGFP-tagged Eps15-D3 $\Delta 2$ had no effect on Tfn uptake. Similar results were observed for Fc $\alpha$ R endocy- 
A

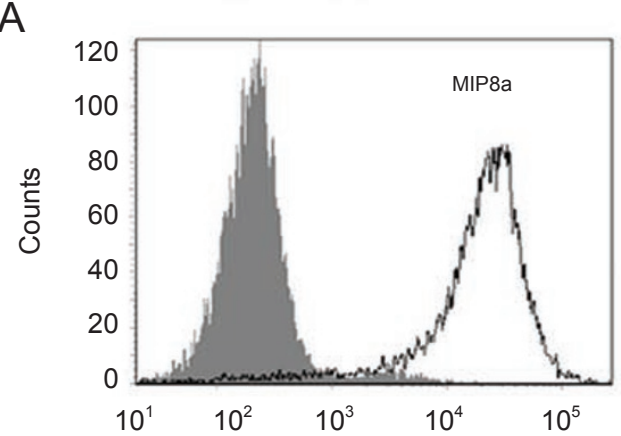

B

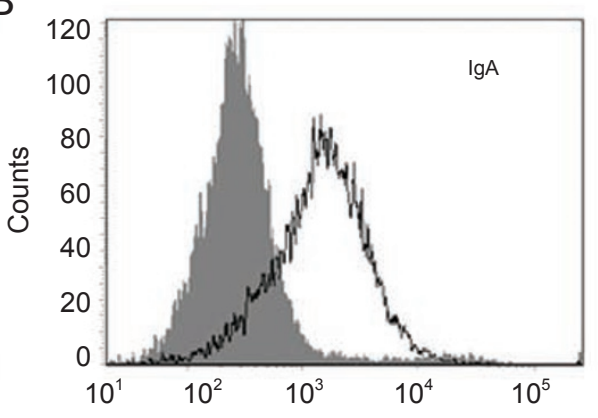

C

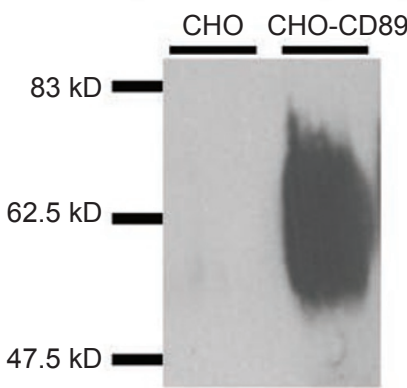

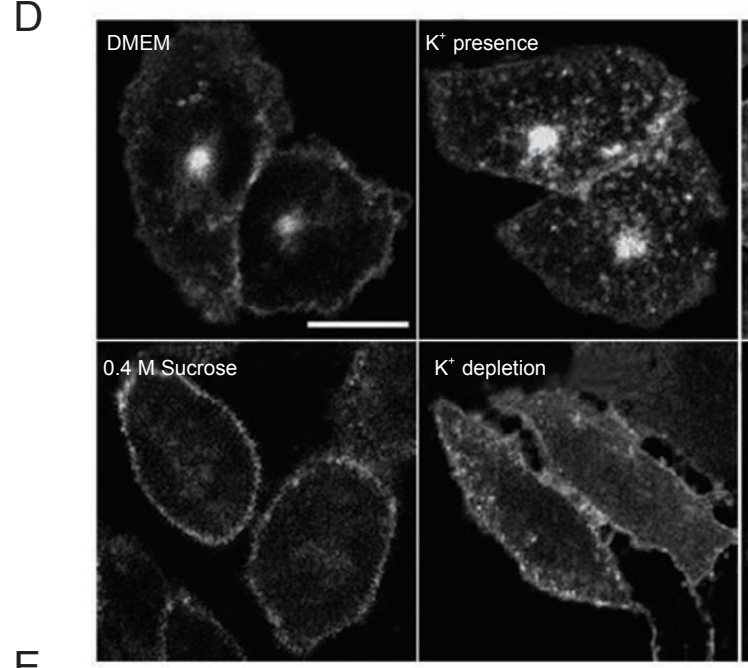

E

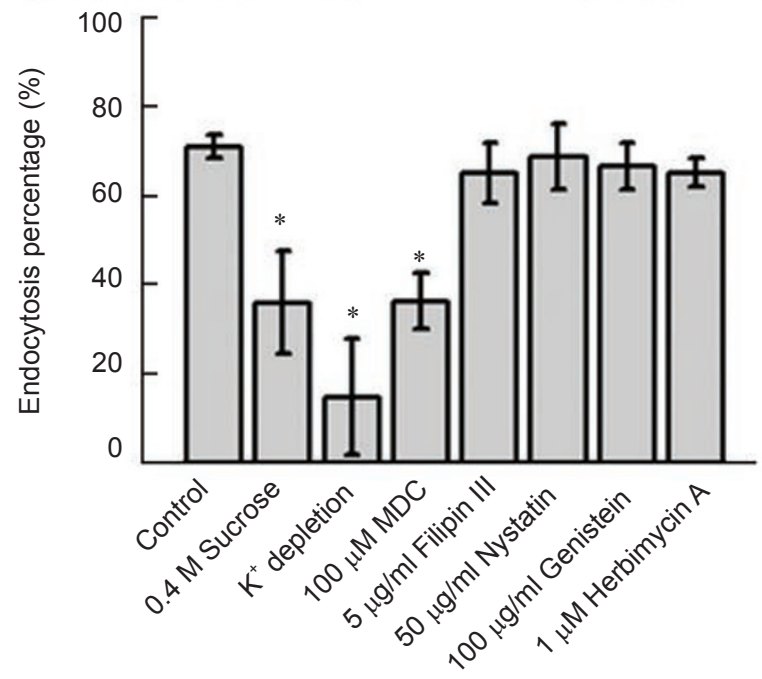

Figure 2 Endocytosis of FcaR in stably transfected $\mathrm{CHO}$ cells. (A) $\mathrm{CHO}$ cells stably expressing FcaR were incubated with MIP8a (black line) or isotype control MOPC21 (filled) for 60 min on ice, followed by incubation with FITC-conjugated goat anti-mouse IgG. (B) $\mathrm{CHO}$ cells (filled) or $\mathrm{CHO}$ cells stably expressing FcaR (black line) were incubated with FITC-conjugated human IgA2 for $60 \mathrm{~min}$ on ice. (C) $5 \times 10^{6}$ $\mathrm{CHO}$ cells or $\mathrm{CHO}$ cells stably expressing FcaR were lysed, FcaR were purified by anti-FcaR mAb-coupled beads and analyzed by western blot. (D) Chemical inhibitor tests were done the in the same manner as that in U937 cells, and the endocytosis was examined by confocal laser-scanning microscope. Bar represents $10 \mu \mathrm{m}$. Data are representative of at least three independent experiments. (E) Quantitative analysis of the effect of chemical inhibitors on FcaR endocytosis by flow cytometry, see Materials and Methods. $* P<$ 0.05 .

tosis. Both Eps15-EH29 and Eps15-DIII but not Eps15D $3 \Delta 2$ inhibited internalization of Fc $\alpha$ R (Figures $3 \mathrm{C}$ and $3 \mathrm{D}$ ), indicating that $\mathrm{Fc} \alpha \mathrm{R}$ was internalized via clathrinmediated endocytosis.

FcaR endocytosis is abolished when clathrin heavy chain is depleted by short-hairpin (shRNA)

The above mentioned inhibition experiments using

chemical inhibitors and dominant-negative mutants of Eps15 indicated that endocytosis of $F c \alpha R$ was dependent on clathrin. To obtain direct evidence, RNA interference was used to knockdown clathrin heavy chain (CHC). We first tried to knockdown $\mathrm{CHC}$ in $\mathrm{CHO}$ cells, but failed (data not shown). Considering that our target sequence (GTA ATC CAA TTC GAA GAC C) against $\mathrm{CHC}$ is only conserved in humans and mice, but not in 
A
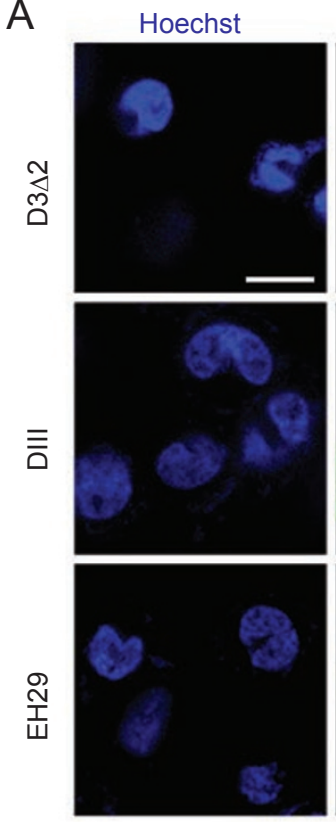

B

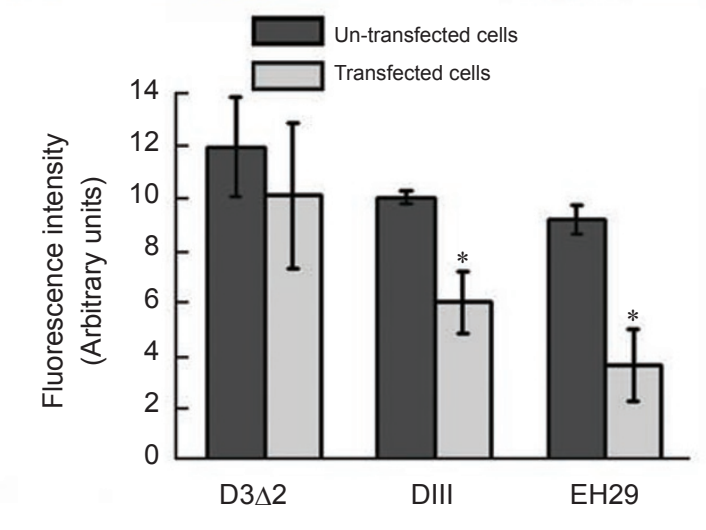

GFP
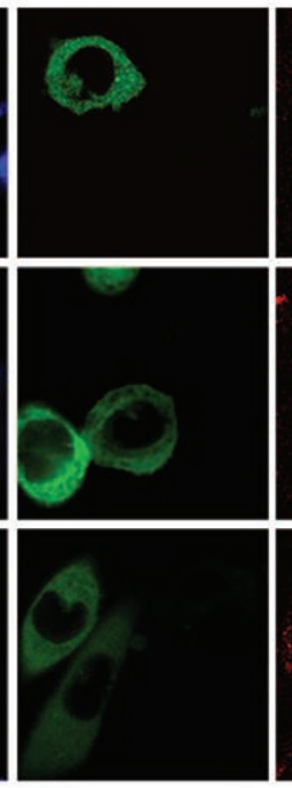
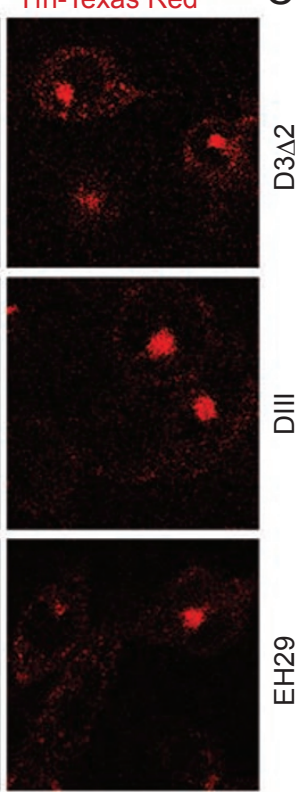

กิ
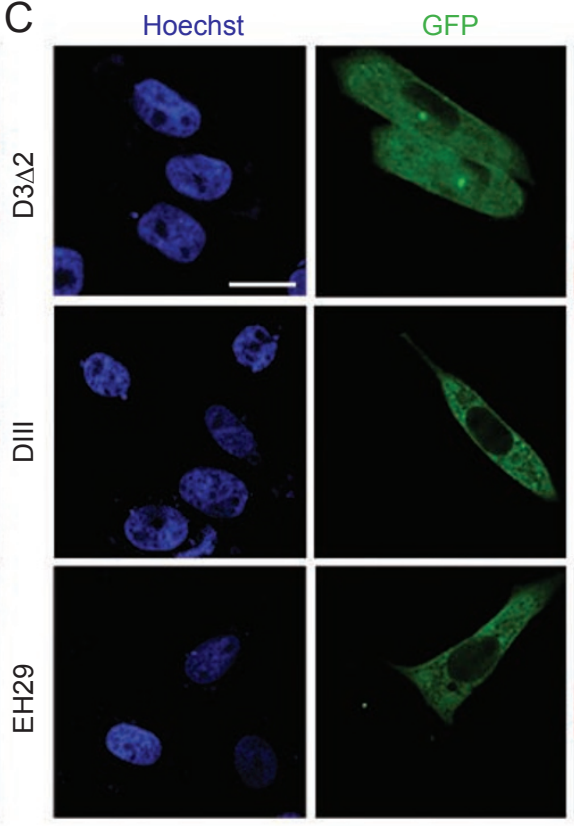

D

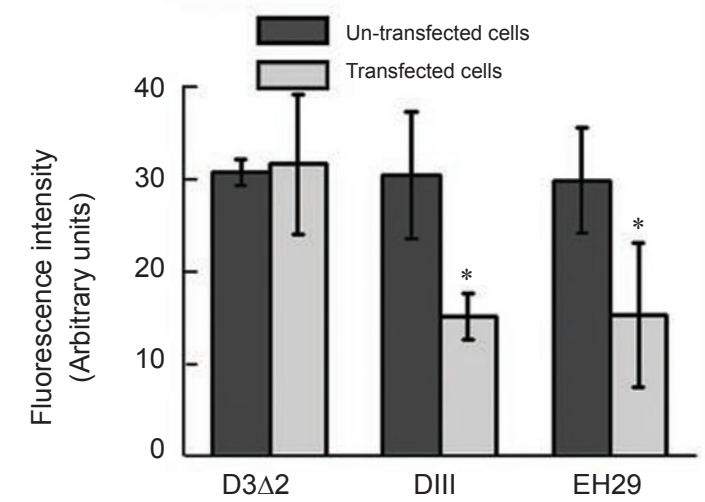

MIp8a-TRITC
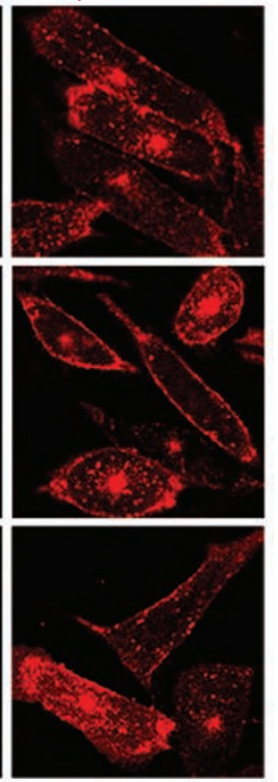

\section{B}

Figure 3 Overexpression of dominant-negative mutants of Eps15 inhibited FcaR endocytosis. CHO cells stably expressing FcaR were transiently transfected with EGFP-tagged Eps15-D3 $\Delta 2$ (WT control), Eps15-DIII or Eps15-EH29. At 48 h posttransfection, cells were allowed to internalize TRITC-conjugated MIP8a-F(ab') for 30 min at $37^{\circ} \mathrm{C}(\mathrm{C})$. For transferrin uptake (A), cells were serum starved for $1 \mathrm{~h}$ at $37^{\circ} \mathrm{C}$, and then incubated with $50 \mu \mathrm{g} / \mathrm{ml}$ Texas Red-Tfn in DMEM without serum at $37{ }^{\circ} \mathrm{C}$ for $30 \mathrm{~min}$. At the end of endocytosis, cells were cooled to $4{ }^{\circ} \mathrm{C}$ quickly, washed and fixed. Nuclei were stained with Hoechst 33258 (blue). Endocytosis was analyzed by confocal laser-scanning microscope. (B) and (D) are quantitative analysis for Tfn uptake and FcaR endocytosis, respectively. ${ }^{*} P<0.01$. Bars represent $10 \mu \mathrm{m}$. Data are representative of at least three independent experiments.

rats, we speculated that this target sequence might not be conserved in hamster-originated $\mathrm{CHO}$ cells either. So, we used human-originated Hela cells instead. Fc $\alpha$ R was cotransfected with pSUPER vector (mock control) or pSUPER-shRNA CHC in Hela cells, and then endocytosis of $\mathrm{Fc} \alpha \mathrm{R}$ was examined. The expression level of clathrin in Hela cells was obviously decreased $72 \mathrm{~h}$ posttransfection of pSUPER-shRNA CHC, as determined by western blot (Figures 4A and 4B). Immunofluorescent staining of CHC also showed that $\mathrm{CHC}$ was successfully depleted in pSUPER-shRNA CHC-transfected cells, but not in pSUPER-transfected cells (Figure 4C). As shown in Figures $4 \mathrm{C}$ and $4 \mathrm{D}, \mathrm{Fc} \alpha \mathrm{R}$ was able to be internalized when cells were cotransfected with pSUPER vector, but the endocytosis was abolished when pSUPER-shRNA $\mathrm{CHC}$ was cotransfected. These results demonstrated that Fc $\alpha$ R was internalized via a clathrin-dependant pathway in Hela cells.

To further investigate whether clathrin is required for endocytosis of $\mathrm{Fc} \alpha \mathrm{R}$ in U937 cells, which naturally express this receptor, we used a lentiviral shRNA vector pLVTHM to knockdown CHC in U937 cells. U937 
A

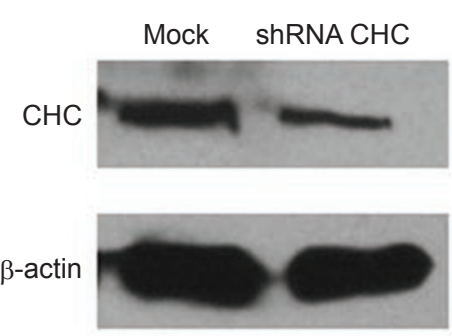

C

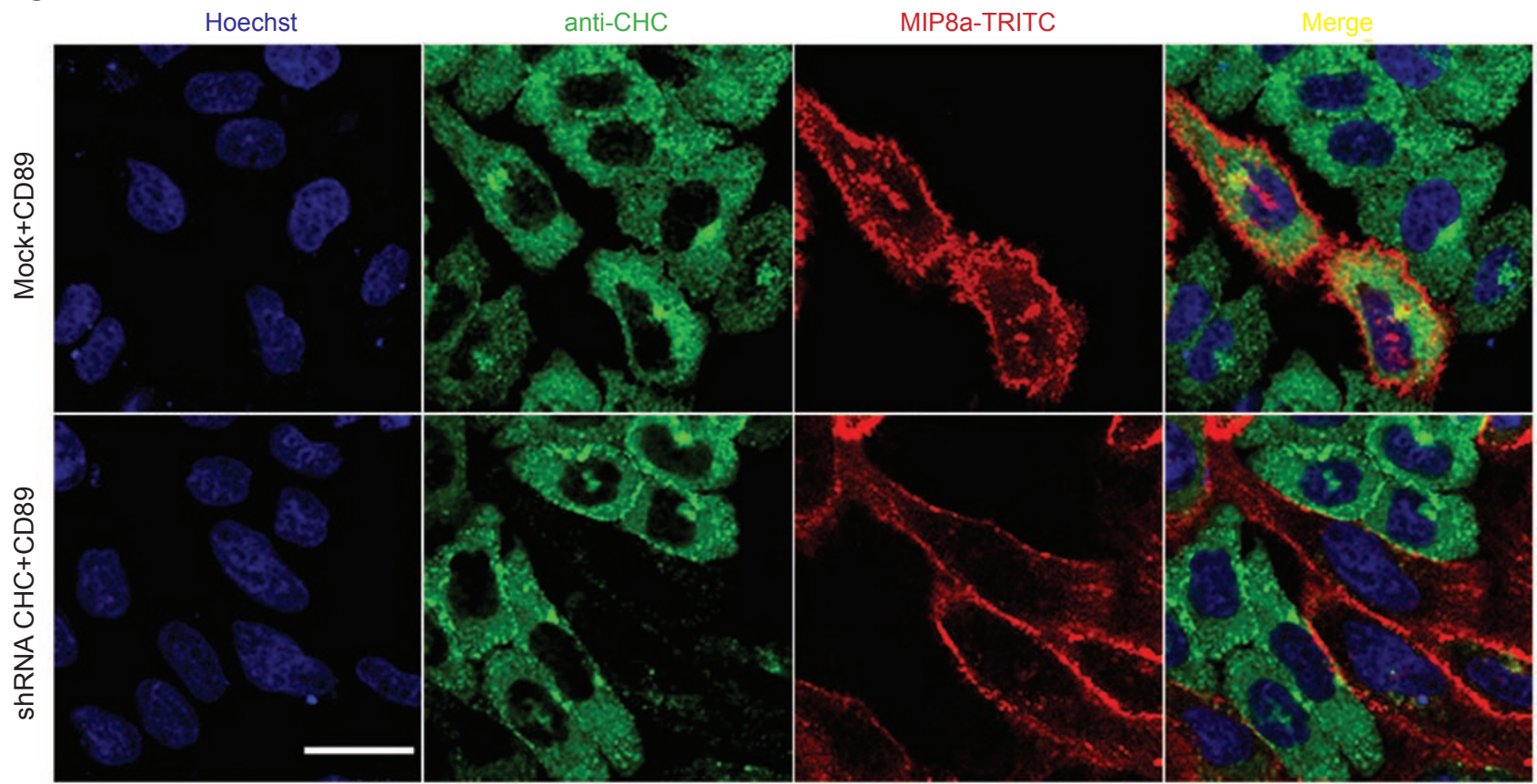

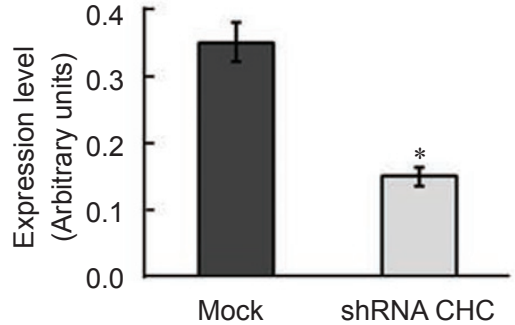

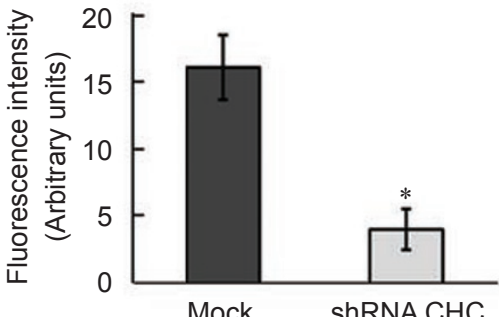

Figure 4 Depletion of clathrin heavy chain by shRNA abolished the endocytosis of FcaR in Hela cells. (A) CHC depletion was analyzed by western blot. Hela cells were transfected with pSUPER-shRNA CHC. At $72 \mathrm{~h}$ posttransfection, equal numbers of cells were lysed, separated by $5 \%-18 \%$ SDS-PAGE and transferred onto a nitrocellulose membrane. CHC was detected by mouse monoclonal Abs TD-1. (B) Relative expression of $\mathrm{CHC}$ was quantified by densitometry. Data are from three independent experiments. ${ }^{*} P<0.01$. (C) Endocytosis of FcaR in Hela cells cotransfected with pcDNA3.1-FcaR and pSUPER-shRNA $\mathrm{CHC}$. At $72 \mathrm{~h}$ posttransfection, cells were allowed to internalize TRITC-conjugated MIP8a-F(ab') ${ }_{2}$ (red) for $30 \mathrm{~min}$ at $37^{\circ} \mathrm{C}$. At the end of endocytosis, cells were fixed, permeabilized and $\mathrm{CHC}$ was stained by rabbit anti-clathrin heavy chain polyclonal Abs followed by FITC-conjugated goat anti-rabbit IgG. Nuclei were stained with Hoechst 33258 (blue). Endocytosis of FcaR was examined by confocal laser-scanning microscope. Bar represents $10 \mu \mathrm{m}$. Data are representative of three independent experiments. (D) Quantitative analysis for FcaR endocytosis after depletion of CHC. ${ }^{*} P<0.01$.

cells were infected with viruses packed in $293 \mathrm{~T}$ cells, and cells with high expression level of GFP were sorted by FACS. Western blot showed that $\mathrm{CHC}$ expression in shRNA CHC U937 cells decreased to $18 \%$ of mock shRNA cells (Figure 5A), which was in line with the result of immunofluorescent staining of $\mathrm{CHC}$, showing that $\mathrm{CHC}$ staining in $\mathrm{CHC}$ knockdown cells was significantly weaker than in control cells (Figure 5C). Then, endocytosis of FcaR in CHC knockdown U937 cells was examined. As shown in Figure 5D, Fc $\alpha \mathrm{R}$ endocytosis in CHC knockdown cells decreased remarkably. Quantitative analysis by flow cytometry showed that endocytosis of Fc $\alpha \mathrm{R}$ in CHC knockdown U937 cells decreased to $28 \%$ of control (Figure 5B), demonstrating that endocytosis of FcaR in U937 cells was clathrin dependent.

Endocytosed FcaR is localized in Rab5- and Rab11-positive endosomes, but endocytosis of FcaR is not regulated by Rab5

Small GTPases broadly regulate endocytosis, intracellular trafficking and membrane fusion of endocytic vesicles. Rab5, Rab11, Rab7 and Rab9 are recognized as 
A
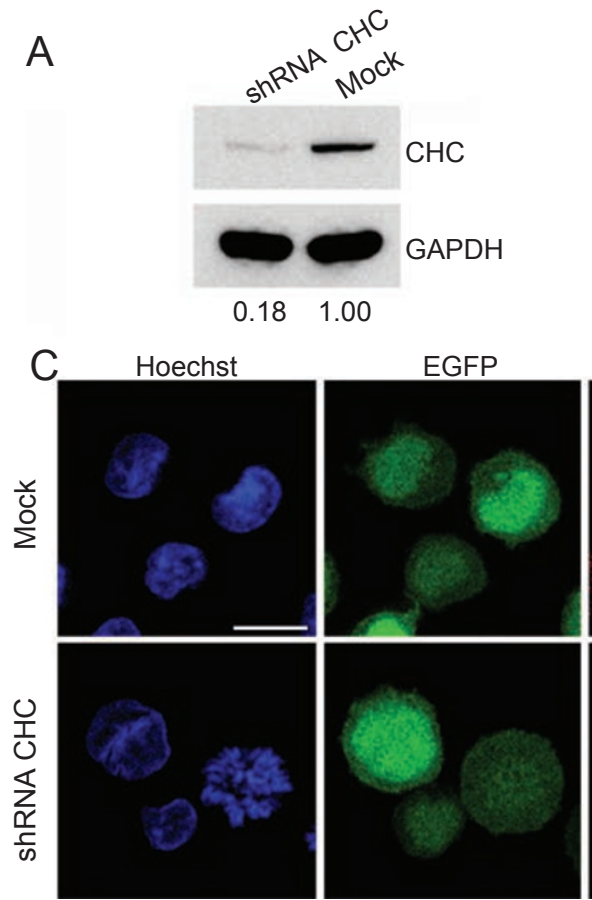
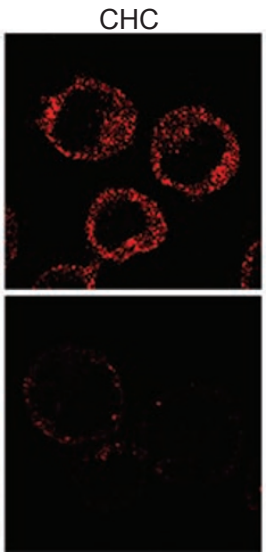
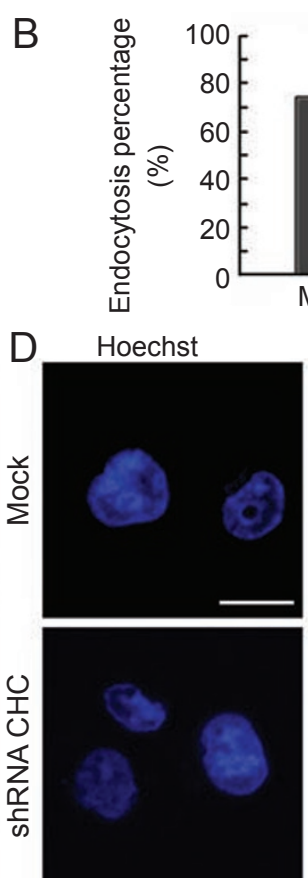

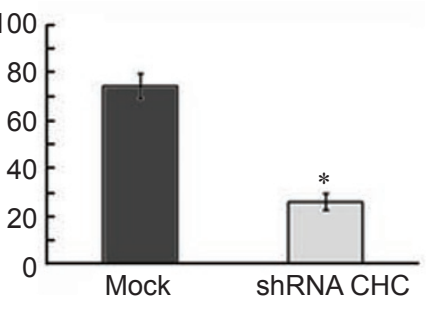

EGFP
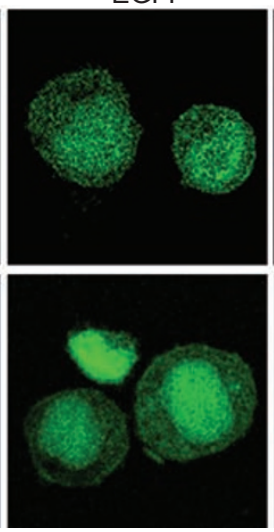

MIP8a-TRITC + Hoechst

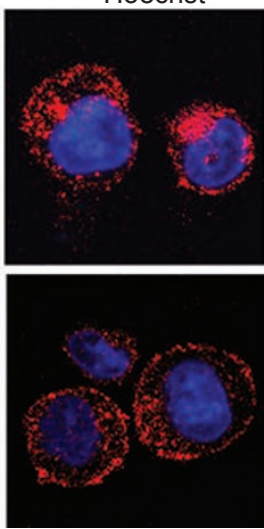

Figure 5 Knockdown of CHC in U937 cells inhibits FcaR endocytosis. (A) CHC expression in U937 cells stably infected with pLVTHM or pLVTHM-shRNA CHC was analyzed by western blot. A total of $30 \mu \mathrm{g}$ protein from each cell lysate was separated by $5 \%-18 \%$ SDS-PAGE, transferred onto a nitrocellulose membrane and $\mathrm{CHC}$ was detected by mouse monoclonal Abs TD-1. The number under each lane is the relative expression level of $\mathrm{CHC}$ quantified by densitometry. (B) Quantification of FcaR endocytosis in pLVTHM and pLVTHM-shRNA CHC cells by flow cytometry. Data are from three independent experiments. ${ }^{*} P$ $<0.01$. (C) Examination of CHC expression in U937 cells stably infected with pLVTHM or pLVTHM-shRNA CHC was by confocal laser-scanning microscope. Cells grew on coverslip were fixed, permeabilized, and stained by rabbit anti-clathrin heavy chain polyclonal Abs followed by FITC-conjugated goat anti-rabbit IgG. Nuclei were stained by Hoechst 33258 (blue). (D) Endocytosis of FcaR in U937 cells stably infected with pLVTHM or pLVTHM-shRNA CHC. Cells were incubated with TRITCconjugated MIP8a-F $\left(\mathrm{ab}^{\prime}\right)_{2}$ for 60 min at $4{ }^{\circ} \mathrm{C}$, washed with cold $\mathrm{HBSS}$ and then incubated at $37^{\circ} \mathrm{C}$ for 30 min to allow endocytosis. After endocytosis, cells were fixed and nuclei were stained by Hoechst 33258 (blue). Bar represents $15 \mu \mathrm{m}$. Data are representative of four independent experiments.

markers for early endosomes, recycling endosomes and late endosomes. We examined whether FcaR was present in these small GTPase-containing endosomes during endocytosis and intracellular trafficking. To visualize the colocalization of Fc $\alpha \mathrm{R}$ with these Rab proteins, $\mathrm{CHO}$ cells stably expressing Fc $\alpha$ R were transiently transfected with plasmids encoding EGFP-tagged Rab5, Rab11, Rab7 or Rab9. At $48 \mathrm{~h}$ posttransfection, FcaR expressed on the cell surface was labeled with TRITC-conjugated MIP8a-F $\left(\mathrm{ab}^{\prime}\right)_{2}$, a monoclonal antibody $(\mathrm{mAb})$ specifically against FcaR. Then cells were incubated at $37{ }^{\circ} \mathrm{C}$ to allow for endocytosis. Localization of internalized FcaR and EGFP-Rab proteins was determined by confocal microscopy. Figure 6A shows that internalized FcaR colocalized with Rab5 and Rab11 30 min after endocytosis. Analysis of the time course of colocalization showed that FcaR colocalized with Rab5 as early as 5 min after the cells were incubated at $37{ }^{\circ} \mathrm{C}$ (Supplementary in- formation, Figure S1A). This colocalization reached a maximum at 30 min and then decreased slowly. Colocalization of FcaR with Rab11 was observed at 10 min and reached a maximum at $60 \mathrm{~min}$, then decreased through the following $3 \mathrm{~h}$ (Supplementary information, Figure $\mathrm{S} 1 \mathrm{~B})$. In comparison, little colocalization between Fc $\alpha \mathrm{R}$ and Rab7 or Rab9 was observed through the whole time course (Supplementary information, Figure S2). These data suggest that internalized Fc $\alpha$ R went into Rab5positive early endosomes, then were possibly recycled back to the cell surface through Rab11-positive recycling endosomes, but not delivered to late endosomes.

Rab5-S34N is a dominant-negative mutant of Rab5, which has been shown to be able to inhibit endocytosis of some receptors [33]. Since Fc $\alpha \mathrm{R}$ was colocalized with Rab5, we next examined whether the endocytosis of $\mathrm{Fc} \alpha \mathrm{R}$ was regulated by Rab5. At $48 \mathrm{~h}$ posttransfection of EGFP-tagged Rab5-S34N in CHO cells stably ex- 


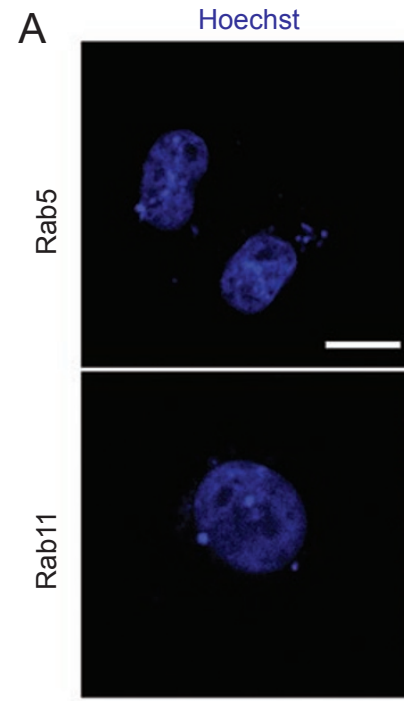

B

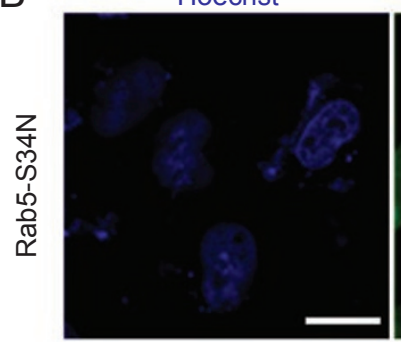

MIP8a-TRITC
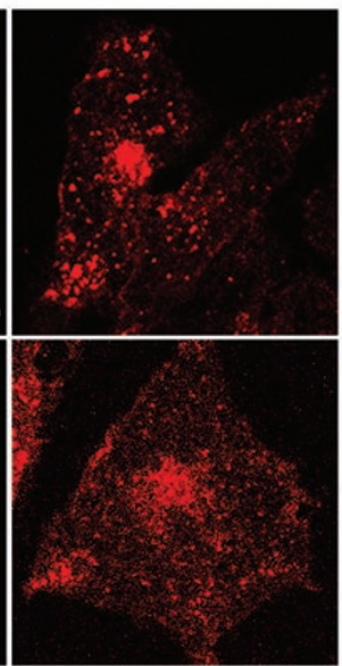

EGFP

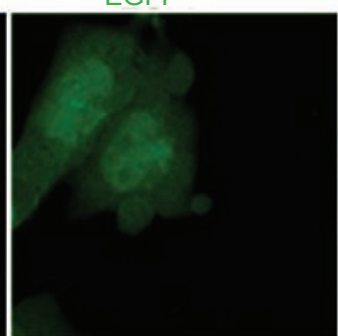

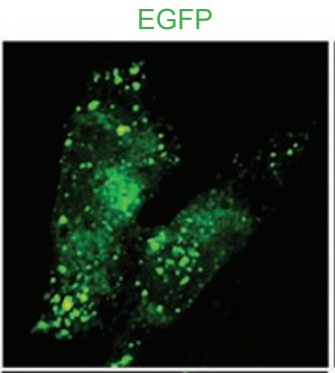

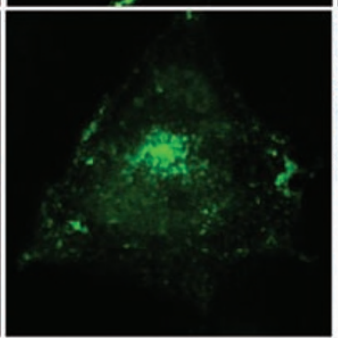

MIP8a-TRITC

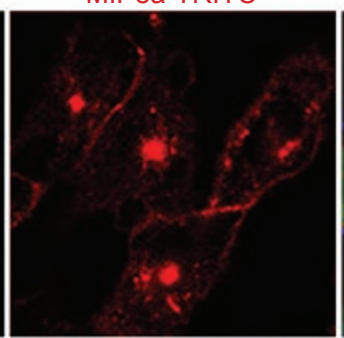

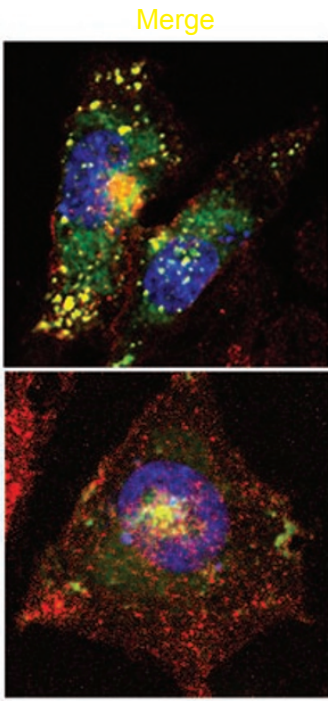

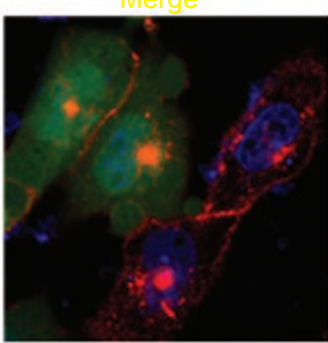

Figure 6 Colocalization of endocytosed FcaR with Rab5 and Rab11. CHO cells stably expressing FcaR were transiently transfected with EGFP-tagged Rab5, Rab11 or Rab5-S34N. At $48 \mathrm{~h}$ posttransfection, cells were incubated with TRITCconjugated MIP8a-F(ab') for 60 min at $4{ }^{\circ} \mathrm{C}$. Excess ligands were washed and cells were transferred to pre-warmed medium $\left(37^{\circ} \mathrm{C}\right)$ to allow for endocytosis for $30 \mathrm{~min}$. Nuclei were stained by Hoechst 33258 (blue). Endocytosis of FcaR was examined by confocal laser-scanning microscope. (A) Colocalization of internalized TRITC-conjugated MIP8a-F(ab') $)_{2}$ with Rab5 (upper panel) or Rab11 (lower panel). (B) Effect of Rab5-S34N on FcaR endocytosis. Bars represent $10 \mu \mathrm{m}$. Data are representative of at least three independent experiments.

pressing $\mathrm{Fc} \alpha \mathrm{R}$, cells were allowed to internalize TRITCconjugated MIP8a-F $\left(\mathrm{ab}^{\prime}\right)_{2}$ for $30 \mathrm{~min}$ at $37^{\circ} \mathrm{C}$. As shown in Figure 6B, overexpression of dominant-negative mutant of Rab5 did not block the internalization of $F c \alpha R$ in $\mathrm{CHO}$ cells, suggesting that the endocytosis of $F c \alpha R$ was not regulated by Rab5.

\section{FcaR endocytosis is dynamin dependent}

Clathrin-dependent and a subset of clathrin-independent endocytosis require the activity of dynamin, a GTPase responsible for pinching vesicles from the plasma membrane and thereby driving cargo internalization into carrier vesicles [17-19]. To determine if Fc $\alpha \mathrm{R}$ internalization was dynamin dependent, HA-tagged WT dynamin (Dyn-WT-HA) or a dominant-negative dynamin mutant K44A (Dyn-K44A-HA) was transiently transfected into CHO cells stably expressing Fc $\alpha$ R. Similar to Tfn (Figures 7A and 7B), FcaR endocytosis was dramatically reduced in cells expressing dominant-negative dynamin mutant, but not in cells expressing the WT dynamin (Figures $7 \mathrm{C}$ and $7 \mathrm{D}$ ), suggesting that dynamin was required for Fc $\alpha \mathrm{R}$ endocytosis.

The endocytic motif of $F c \alpha R$ is not located in the cytoplasmic domain of $F c \alpha R$

Sorting of transmembrane proteins to endosomes is mediated by consensus motifs present within the cytoplasmic domains of these proteins, the most common endocytic motifs are tyrosine-based motif YXXØ (X stands for any amino acid and $\varnothing$ stands for an amino acid residue with a bulky hydrophobic side chain) and dileucinebased signals [DE]XXXL[LI] or DXXLL [44]. As no such conserved motifs are found within the cytoplasmic domain of $F c \alpha R$, we speculate that some unrecognized motif within the cytoplasmic domain might be responsible for Fc $\alpha \mathrm{R}$ endocytosis. To test this hypothesis, the entire cytoplasmic domain of Fc $\alpha \mathrm{R}$ (41 amino acids) was deleted. This tail-less Fc $\alpha \mathrm{R}$ was transiently transfected 

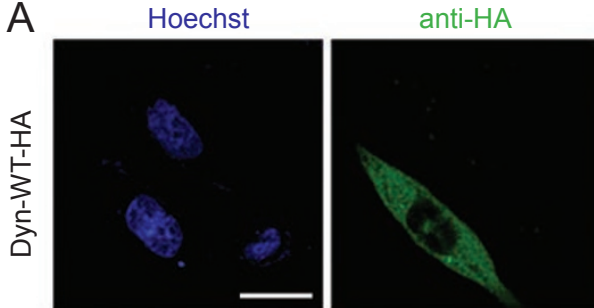

Tfn-Texas Red
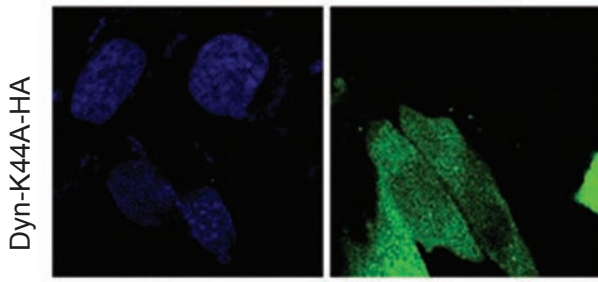

C

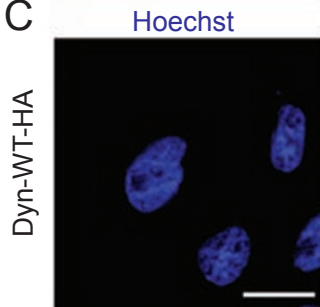

anti-HA
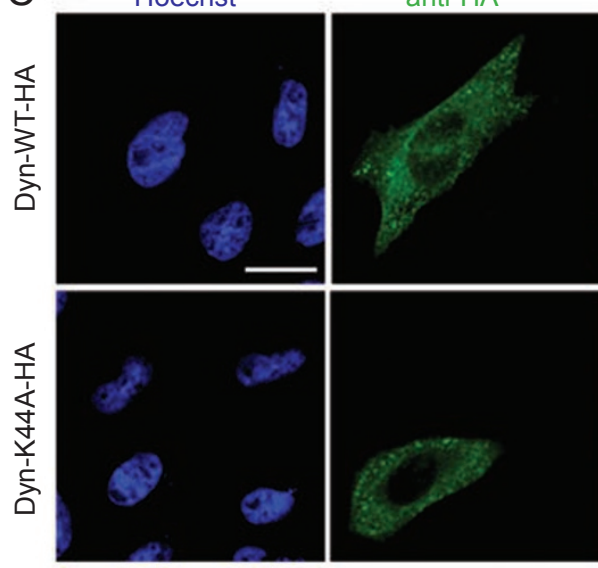
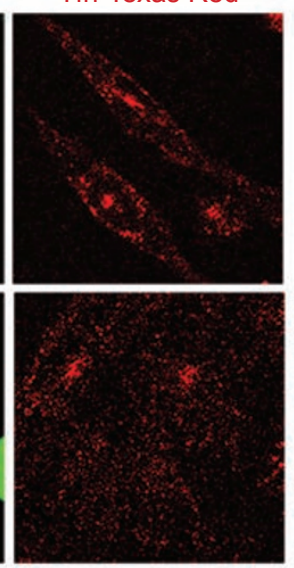

MIP-8a-TRITC

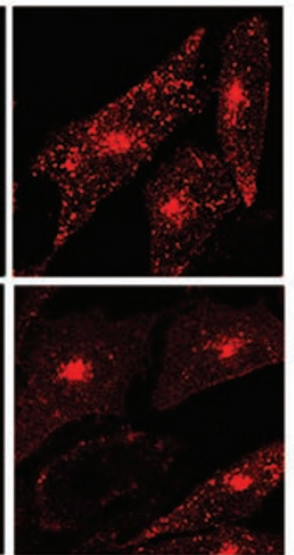

B
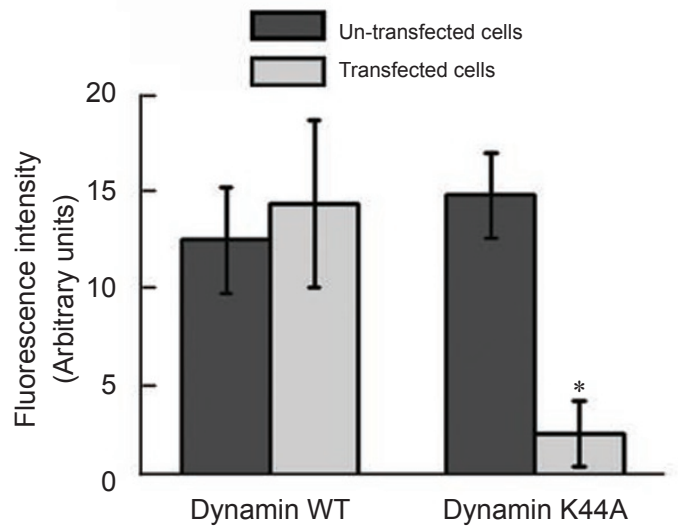

$\mathrm{D}$

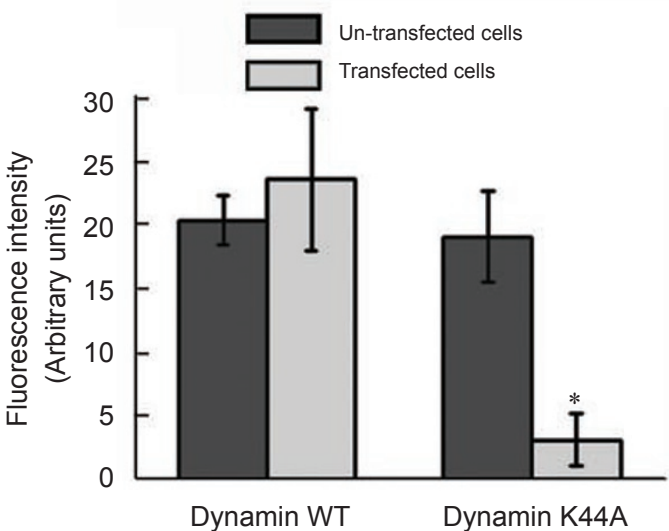

Figure 7 FcaR endocytosis is dynamin dependent. CHO cells stably expressing FcaR were transiently transfected with HAtagged wild-type dynamin (Dyn-WT-HA) or dominant-negative mutant dynamin K44A (Dyn-K44A-HA). At 48 h posttransfection, cells were allowed to internalize TRITC-conjugated MIP8a-F $\left(a b^{\prime}\right)_{2}$ for 30 min at $37^{\circ} \mathrm{C}(\mathbf{C})$. For Tfn uptake $(\mathbf{A})$, cells were serum starved for $1 \mathrm{~h}$ at $37{ }^{\circ} \mathrm{C}$, then incubated with $50 \mu \mathrm{g} / \mathrm{ml}$ Texas Red-Tfn in DMEM without serum at $37^{\circ} \mathrm{C}$ for $30 \mathrm{~min}$. At the end of endocytosis, cells were cooled to $4{ }^{\circ} \mathrm{C}$ quickly, washed, fixed and nuclei were stained with Hoechst 33258 (blue). Endocytosis was analyzed by confocal laser-scanning microscope. (B) and (D) are quantitative analysis for Tfn uptake and FcaR endocytosis, respectively. $* P<0.01$. Bars represent $10 \mu \mathrm{m}$. Data are representative of at least three independent experiments.

in COS-7 cells and stably transfected in $\mathrm{CHO}$ cells. Flow cytometry analysis showed that this tail-less $F c \alpha R$ was able to be expressed on cell surface, and bind IgA to the same extent as the full-length receptor (data not shown). Endocytosis results showed that, just like the full-length receptor, the tail-less FcaR was able to be internalized both in COS-7 (Figure 8A) and CHO cells (Figure 8B).

To test whether this tail-less Fc $\alpha \mathrm{R}$ is internalized through the same pathway as the full-length receptor, chemical inhibitors of clathrin- or caveolae/lipid raftmediated endocytosis pathway were used. As shown in Figures $8 \mathrm{C}$ and $8 \mathrm{E}$, hypertonic sucrose $(0.4 \mathrm{M})$ and MDC $(100 \mu \mathrm{M})$, but not Filipin III $(5 \mu \mathrm{g} / \mathrm{ml})$, inhibited endocytosis of tail-less Fc $\alpha \mathrm{R}$, suggesting that this tail-less
Fc $\alpha \mathrm{R}$ was also endocytosed through a clathrin-dependent pathway. Next, tail-less Fc $\alpha$ R was cotransfected with pSUPER or pSUPER-shRNA CHC in Hela cells, as done for full-length FcaR. As expected, CHC was depleted in pSUPER-shRNA CHC-transfected cells, but not in pSUPER-transfected cells. In addition, endocytosis of tail-less FcaR in CHC knockdown Hela cells was also inhibited (Figures 8D and 8F). These data demonstrated that endocytosis of tail-less FcaR was, like full-length Fc $\alpha$ R, also through the clathrin-dependent pathway.

We also compared the kinetics of endocytosis of FcaR and tail-less FcaR in stably transfected $\mathrm{CHO}$ cells, and the result showed no difference between them (Figure $8 \mathrm{E})$. These data suggest that endocytosis of both full- 

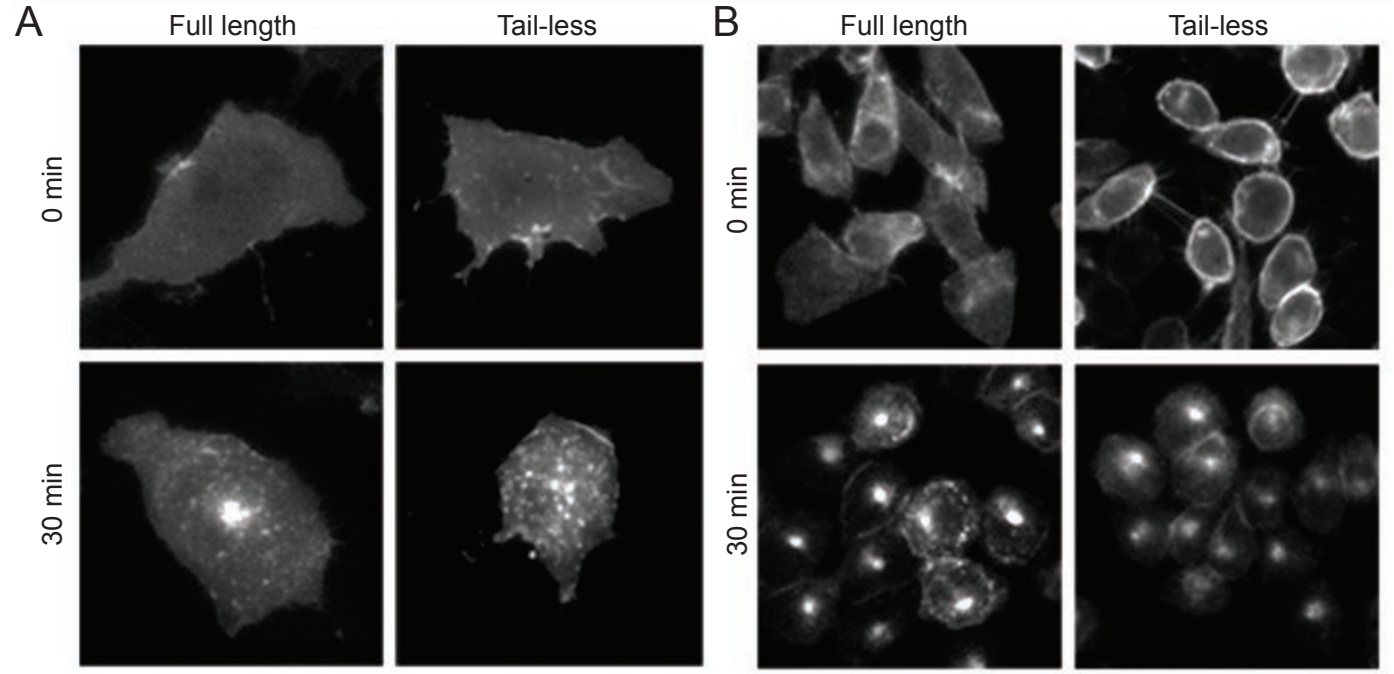

C

Control

0.4 M Sucrose
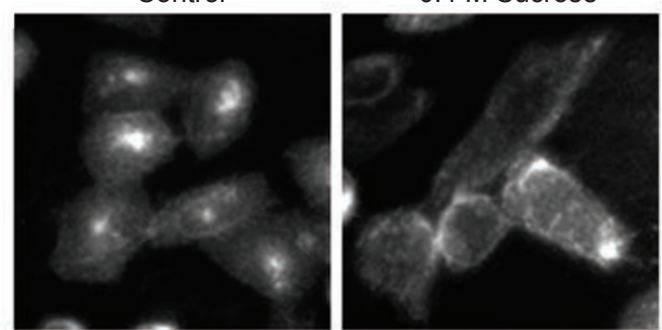

$100 \mu \mathrm{M}$ MDC

$5 \mu \mathrm{g} / \mathrm{ml}$ Filipin III

D

Hoechst

$\mathrm{CHC}$

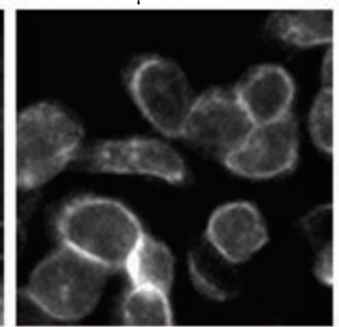

MIP8a-TRITC
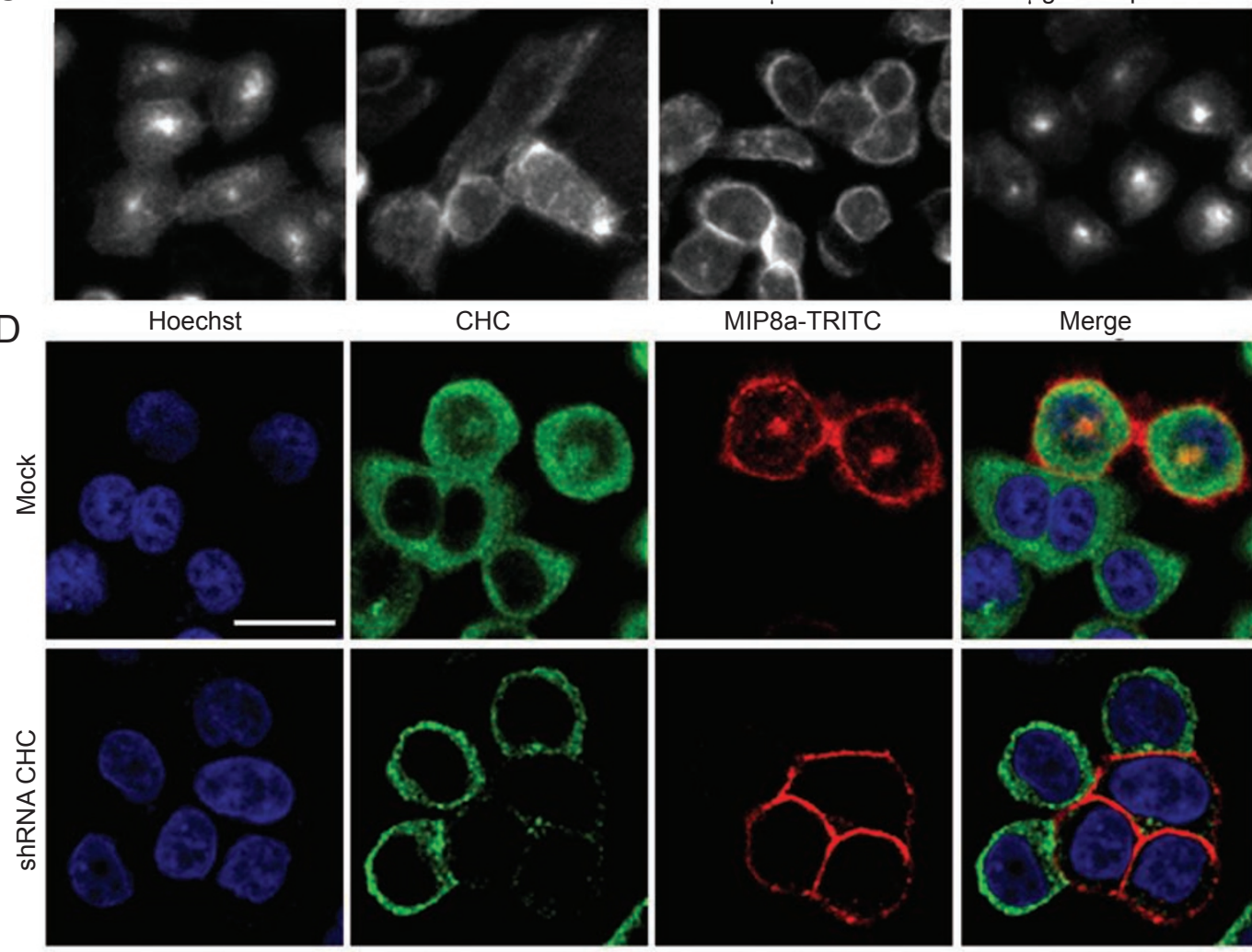

E

- - Full length

F

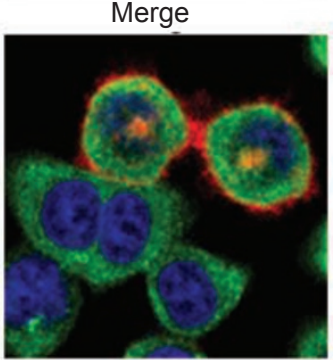

-O. Tail-less
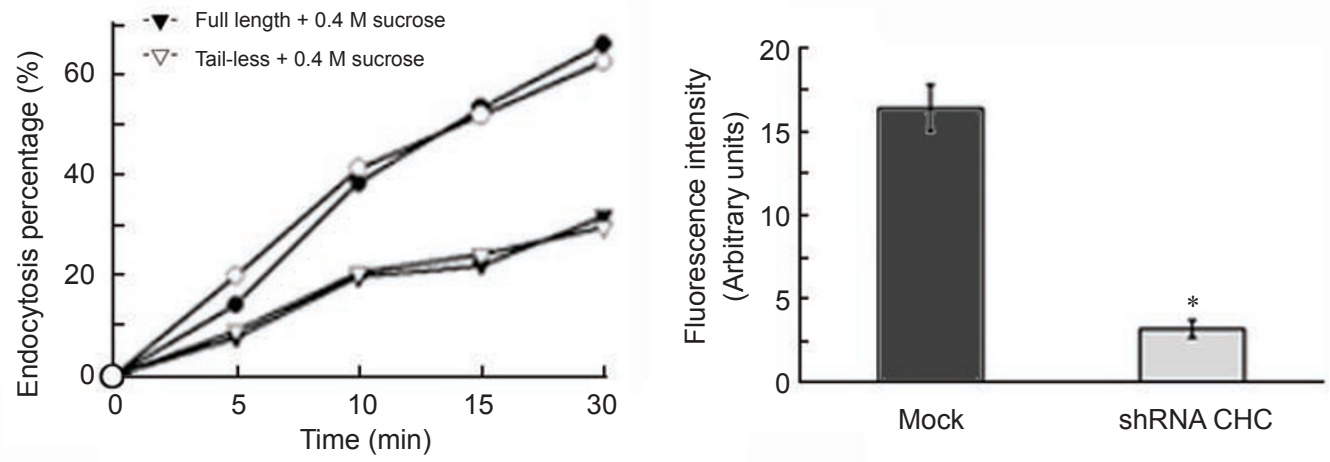

Cell Research | Vol 20 No 2 | February 2010 
length $F c \alpha R$ and tail-less Fc $\alpha$ R follows the same clathrin-dependent pathway, but the cytoplasmic domain is not required for endocytosis of $\mathrm{Fc} \alpha \mathrm{R}$.

\section{Discussion}

Although endocytosis is a well-known feature of $\mathrm{Fc} \alpha \mathrm{R}$, the mechanism remains unknown. Previous studies showed that $F c \alpha R$ redistributed into lipid raft $30 \mathrm{~s}$ after being cross-linked, but this redistribution decreased to a basal level within $5 \mathrm{~min}$ before endocytosis occurred $[9,10]$. In addition, FcaR was not colocolized with the lipid raft marker, GM-1 [10]. These results suggested that endocytosis of FcaR was not lipid raft mediated. In the present study, we investigated the Fc $\alpha$ R endocytic pathway and demonstrated that $\mathrm{Fc} \alpha \mathrm{R}$ was internalized through a clathrin- and dynamin-dependent pathway.

Endocytosis of FcaR was blocked by hypertonic sucrose, MDC and $\mathrm{K}^{+}$depletion, but not by nystatin and Filipin III, suggesting that $F c \alpha R$ underwent endocytosis via a clathrin-dependent pathway, but not the caveolae or lipid raft-mediated endocytosis. Furthermore, tyrosine kinases were not required for Fc $\alpha \mathrm{R}$ endocytosis, as genistein and herbimycin A did not inhibit the endocytosis of Fc $\alpha$ R. This is very similar to Fc $\gamma \mathrm{RI}$ (CD64), which can undergo endocytosis in the absence of $\gamma$-chain in a tyrosine kinase-independent manner [45]. In comparison, tyrosine kinase is required for the endocytosis of FcyRIIA [46]. These data suggest that endocytosis of different $\mathrm{Fc}$ receptors is differentially regulated by tyrosine kinase.

To validate the results of chemical inhibitor experiments, dominant-negative mutants of Eps15 were used, which can specifically inhibit clathrin-mediated endocytosis [43]. As shown in Figure 3, expression of both mutants, but not WT control Eps15, blocked the endocytosis of FcaR. This further demonstrated that Fc $\alpha R$ was internalized through clathrin-mediated endocytosis. Moreover, Fc $\alpha$ R endocytosis was abolished when clath- rin heavy chain was depleted by shRNA, providing direct evidence that clathrin was required for endocytosis of Fc $\alpha$ R. Taken together, these data demonstrate that endocytosis of FcaR is clathrin-mediated.

$\mathrm{Fc} \alpha \mathrm{R}$ is expressed on various myeloid cells either as monomers or FcR- $\gamma$-chain-associated multimers [12]. Previous studies using IIA1.6 and RBL-2H3 cells showed that FcR $\gamma$-chain was not required for Fc $\alpha$ Rmediated endocytosis of IgA and IgA immune complexes $[9,12]$. Here, we demonstrated that Fc $\alpha \mathrm{R}$ was able to undergo endocytosis in transfected CHO, COS-7 and Hela cells, which are not myeloid cells and devoid of $\gamma$-chain. Therefore, endocytosis is an intrinsic characteristic of $\mathrm{Fc} \alpha \mathrm{R}$ itself in various kinds of cells, regardless of the presence of FcR $\gamma$-chain.

Although FcR $\gamma$-chain is not required for FcaR endocytosis, it may affect the fate of FcaR after it enters the cell. Previous study showed that, in transfected RBL cells, internalized Fc $\alpha$ R-R209L (a mutant Fc $\alpha$ R where arginine 209 at the transmembrane region is replaced by lysine, therefore it cannot associate with the $\gamma$-chain) was colocalized with Tfn [12], a known marker for the endocytosis-recycling route. In agreement with these results, we found that internalized FcaR went into Rab5and Rab11-positive early endosomes and recycling endosomes, and it hardly went to Rab7- and Rab9-positive late endosomes. In contrast, when FcaR was associated with $\gamma$-chain, the endocytosed ligand was sorted to lysosomes for degradation and further for antigen presentation [13]. Therefore, $\gamma$-chain plays important roles in determining the intracellular trafficking routes of $F c \alpha R$ after endocytosis $[12,13]$.

Although Rab5 has been shown to be able to regulate the endocytosis of some membrane receptors [33], overexpression of a dominant-negative mutant of Rab5 (Rab5-S34N) had no influence on the endocytosis of $\mathrm{Fc} \alpha \mathrm{R}$ in this study. This indicates that endocytosis of $\mathrm{Fc} \alpha \mathrm{R}$ is not regulated by Rab5, similar phenomenon have been reported for other receptors $[47,48]$.

Figure 8 The cytoplasmic domain of FcaR is not required for its endocytosis. FcaR and tail-less FcaR were transiently transfected in COS-7 cells (A) or stably transfected in $\mathrm{CHO}$ cells (B). Cells were incubated with FITC-conjugated MIP8a-F(ab') for $60 \mathrm{~min}$ at $4{ }^{\circ} \mathrm{C}$, then washed and transferred quickly to pre-warmed $\left(37^{\circ} \mathrm{C}\right)$ medium and incubated for 30 min to allow for endocytosis. The endocytosis was examined by fluorescence microscope. (C) Endocytosis of tail-less FcaR stably transfected in $\mathrm{CHO}$ cells in the presence of sucrose $(0.4 \mathrm{M})$, MDC $(100 \mu \mathrm{M})$ or Filipin III $(5 \mu \mathrm{g} / \mathrm{ml})$. The endocytosis was examined by fluorescence microscope. (D) Endocytosis of tail-less FcaR in CHC knockdown Hela cells. Hela cells were cotransfected with pcDNA3.1-tail-less FcaR and pSUPER or pSUPER-shRNA CHC. At $72 \mathrm{~h}$ posttransfection, cells were allowed to internalize TRITC-conjugated MIP8a-F $\left(\mathrm{ab}^{\prime}\right)_{2}$ for $30 \mathrm{~min}$ at $37^{\circ} \mathrm{C}$. At the end of endocytosis, cells were fixed, permeabilized and $\mathrm{CHC}$ was stained by rabbit anti-clathrin heavy chain polyclonal Abs followed by FITC-conjugated goat anti-rabbit IgG. Nuclei were stained by Hoechst 33258 (blue). The endocytosis was examined by confocal laser-scanning microscope. Bar represents $15 \mu \mathrm{m}$. Data are representative of three independent experiments. (E) Kinetic analysis of endocytosis of FcaR and tail-less FcaR in stably transfected $\mathrm{CHO}$ cells by flow cytometry. (F) Quantitative analysis of tail-less FcaR endocytosis in CHC knockdown Hela cells. $* P<0.01$. 
It is believed that signals for clathrin-mediated endocytosis lie in the cytoplasmic domain of membrane receptors. Because there are no such conserved endocytic motifs within the cytoplasmic domain of Fc $\alpha R$, we, as well as others [13], speculated that there might be some unrecognized motif within the cytoplasmic domain of Fc $\alpha R$, which could interact with endocytic machinery and mediate endocytosis of FcaR. However, we surprisingly found that $\mathrm{F} c \alpha \mathrm{R}$ could still be internalized when the entire cytoplasmic domain was deleted. What is more interesting is that endocytosis of this tail-less FcaR was inhibited by hypertonic sucrose, MDC and knockdown of $\mathrm{CHC}$, demonstrating that endocytosis of tail-less Fc $\alpha \mathrm{R}$ was also clathrin dependent. Nevertheless, this finding is consistent with the fact that no conserved endocytic motifs are found within the cytoplasmic domain of Fc $\alpha$ R. A few studies showed that signals for endocytosis could localize in the extracellular and/or transmembrane domain of a receptor $[45,49,50]$. For example, it has been reported that endocytic motif of another Fc receptor, Fc $\gamma \mathrm{RI}$ (CD64), localizes within the extracellular domain of the receptor rather than the transmembrane or cytoplasmic domain [45]. How FcaR could be internalized through clathrin-mediated endocytosis in the absence of its cytoplasmic domain is not clear at present.

First, it is possible that the endocytic motif for FcaR might also be localized within the extracellular domain of Fc $\alpha$ R, just like Fc $\gamma R I$. The extracellular region of FcaR contains two Ig-like domains, EC1 and EC2. EC1 contains the binding site for $\operatorname{IgA}$, and our $\mathrm{mAb}$ against $\mathrm{Fc} \alpha \mathrm{R}$ also recognizes EC1. Deletion of EC1 will result in a receptor without the capacity to bind its natural ligand and our $\mathrm{mAb}$ will not recognize it. FcaR without the entire EC2 domain is actually an isoform of Fc $\alpha R$, which is generated through alternative splicing [5]. This isoform of Fc $\alpha \mathrm{R}$ has been only detected at mRNA level, and no surface expression on natural cells has been reported [51]. So, EC2 is important for the surface expression of Fc $\alpha$ R. Therefore, to characterize the exact endocytic motif of FcaR, one would need to make site-directed mutations within its extracellular or transmembrane domain.

Second, we think that characterizing endocytic adaptors of Fc $\alpha \mathrm{R}$ is more important, because wherever the endocytic motif is localized, this motif must interact with the endocytic adaptors to accomplish endocytosis. So, we have tried to identify FcaR-interacting endocytic adaptors (or other transmembrane molecules) by CoIP. However, silver staining generated many weak bands (data not shown), which were difficult to characterize and might reflect non-specific bindings. Other techniques, such as yeast two-hybrid screening, might be able to identify Fc $\alpha$ R-interacting proteins.
In conclusion, we demonstrate that $\mathrm{Fc} \alpha \mathrm{R}$ is able to be internalized through clathrin-mediated endocytosis in the absence of its cytoplasmic domain. Our data shed new light on the mechanism of clathrin-mediated endocytosis by showing that the endocytic motif does not have to be localized in the cytoplasmic domain of the receptor. Further studies are needed for characterizing the exact endocytic motif of Fc $\alpha \mathrm{R}$ and endocytic adaptors that help tailless FcaR accomplish endocytosis. Better understanding of the mechanisms of FcaR endocytosis will help us elucidate the role of this receptor in regulating serum IgA homeostasis and IgA-mediated immune responses.

\section{Materials and Methods}

\section{Antibody and agents}

The murine anti-Fc $\alpha \mathrm{R}$ mAb MIP8a, rabbit anti-Fc $\alpha \mathrm{R}$ polyclonal antibody, FITC or tetramethylrhodamine isothiocyanate (TRITC, USA)-labeled $\mathrm{F}\left(\mathrm{ab}^{\prime}\right)_{2}$ fragments of MIP8a and human IgA2 were prepared as described previously [52-54]. Rabbit anticlathrin heavy chain (CHC) polyclonal antibody (ab21679) was from Abcam (Cambridge, UK), mouse anti-CHC mAb (TD-1) was from Santa Cruz Biotechnology (Santa Cruz, USA). Goat anti-HA polyclonal antibody, HRP-conjugated goat anti-mouse IgG polyclonal antibody and FITC-conjugated goat anti-rabbit IgG polyclonal antibody were from Zhongshan Biotechnology Co (Beijing, China). Sucrose, monodansylcadaverine crystalline (MDC), Filipin III, nystatin, genistein, herbimycin A and phorbol-12-myristate-13acetate (PMA) were from Sigma-Aldrich (St. Louis, MO, USA). Texas Red-conjugated Tfn was from Invitrogen (Carlsbad, USA).

\section{Plasmids}

The cDNA of FcaR was cloned from human peripheral white blood cells by reverse transcription PCR, and inserted into the BamHI and XhoI sites of pcDNA 3.1 vector (Invitrogen). The tail-less FcaR (the entire cytoplasmic domain of Fc $\alpha \mathrm{R}$ was deleted) was constructed by standard PCR procedure and inserted into pcDNA3.1 vector at the same sites. EGFP-tagged Eps15EH29, DIII and D3 $\Delta 2$ were provided by Dr Alexandre Benmerah [43]. HA-tagged WT dynamin and its dominant-negative mutant K44A were provided by Dr Sandra Schmid [19]. EGFP-tagged Rab7, Rab9 and Rab11 were gifts from Dr Richard E Pagano [55]. EGFP-tagged Rab5 and Rab5-S34N were provided by Dr Feng Du (Tsinghua University, China).

\section{Cell culture and transfection}

U937 cells were grown in RPMI1640 containing 10\% fetal bovine serum, and $\mathrm{CHO}$, COS-7 and Hela cells were grown in Dulbecco's modified Eagle's medium (DMEM) containing 10\% fetal bovine serum and antibiotics, at $37{ }^{\circ} \mathrm{C}$ in a humidified atmosphere of $5 \% \mathrm{CO}_{2}$. Cells were transfected with plasmids encoding of Fc $\alpha$ R using Lipofectamine 2000 (Invitrogen). Stably transfected CHO cells were selected with $800 \mu \mathrm{g} / \mathrm{ml} \mathrm{G} 418$ and were evaluated for receptor expression by flow cytometry and western blot. Cells stably expressing Fc $\alpha$ R were transiently transfected with EGFPtagged WT and dominant-negative mutant of Eps15, HA-tagged dynamin WT and K44A or EGFP-tagged Rab GTPases using Li- 
pofectamine $\mathrm{TM}^{\mathrm{TM}} 2000$.

\section{Manipulation of endocytic pathways by chemical inhibitors}

Because the expression level of FcaR on U937 cells is relatively low, the signal of FcaR staining under laser-scanning confocal microscopy is weak. So, PMA was used to increase the expression level of FcaR [12]. U937 cells that grew on poly-lysinecoated coverslip were treated with $10^{-7} \mathrm{M}$ PMA for $24 \mathrm{~h}$ before experiments. CHO cells stably expressing FcaR were cultured as described above. $24 \mathrm{~h}$ prior to experiments, cells were seeded onto coverslip placed in 24-well plates and cultured for another 24$48 \mathrm{~h}$. On the day of experiment, cells were pretreated with $0.4 \mathrm{M}$ sucrose, $100 \mu \mathrm{M}$ MDC, $5 \mu \mathrm{g} / \mathrm{ml}$ Filipin III, $50 \mu \mathrm{g} / \mathrm{ml}$ nystatin, 100 $\mu \mathrm{g} / \mathrm{ml}$ genistein or $1 \mu \mathrm{M}$ herbimycin A for $1 \mathrm{~h}$ at $37^{\circ} \mathrm{C}$. $\mathrm{K}^{+}$depletion was done according to the procedure of Altankov and Grinnell [36]. Briefly, cells were rinsed once with potassium-free buffer (140 mM NaCl, $20 \mathrm{mM}$ Hepes, $1 \mathrm{mM} \mathrm{CaCl} 2,1 \mathrm{mM} \mathrm{MgCl} 2,1 \mathrm{mg} /$ $\mathrm{ml} \mathrm{D}$-glucose, $\mathrm{pH} 7.4)$, then incubated in hypotonic medium $(50 \%$ potassium-free buffer $/ 50 \% \mathrm{H}_{2} \mathrm{O}$ ) for $5 \mathrm{~min}$ at $37{ }^{\circ} \mathrm{C}$, followed by incubation in potassium-free buffer for $20 \mathrm{~min}$ at $37^{\circ} \mathrm{C}$. Cells were then cooled to $4{ }^{\circ} \mathrm{C}$ and incubated with FITC or TRITClabeled MIP8a-F $\left(\mathrm{ab}^{\prime}\right)_{2}$ in medium or potassium-free buffer at $4{ }^{\circ} \mathrm{C}$ for $30 \mathrm{~min}$. Cells were washed with cold HBSS (or potassium-free buffer), then transferred quickly into pre-warmed $\left(37^{\circ} \mathrm{C}\right)$ medium supplemented with inhibitors or potassium-free buffer, followed by incubation at $37{ }^{\circ} \mathrm{C}$ for various times to allow for endocytosis. Controls constituted of equal volume of respective solvent added in medium. In $\mathrm{K}^{+}$depletion assay, control was conducted with 10 $\mathrm{mM} \mathrm{KCl}$ added in potassium-free buffer. At the end of endocytosis, cells were placed on ice, fixed with $4 \%$ paraformaldehyde and mounted. The inhibition of endocytosis was determined by confocal microscopy and quantified by flow cytometry.

\section{Flow cytometry}

To analyze the expression of Fc $\alpha \mathrm{R}$ on CHO cells, stably transfected cells were incubated with MIP8a or isotype control MOPC21 (Sigma-Aldrich) in 1\% BSA/PBS followed by incubation with FITC-conjugated goat anti-mouse IgG. Then samples were analyzed by a flow cytometer (FACS Aria, USA). For IgA binding, $\mathrm{CHO}$ cells or Fc $\alpha \mathrm{R}$ stably transfected $\mathrm{CHO}$ cells were incubated with FITC-conjugated human IgA2 in 1\% BSA/PBS for $60 \mathrm{~min}$ on ice, then washed and analyzed by flow cytometry.

Endocytosis of Fc $\alpha \mathrm{R}$ in U937 cells and stably transfected $\mathrm{CHO}$ cells was quantified by flow cytometry. Cells were incubated with MIP8a in medium (or medium containing inhibitors) for $60 \mathrm{~min}$ on ice, washed with cold HBSS thrice (for U937 cells, cells were centrifuged at $400 \times g$ for $2 \mathrm{~min}$ after each wash), then cells were incubated in medium (or medium containing inhibitors) at $37{ }^{\circ} \mathrm{C}$ to allow for endocytosis for various times. The control sample was placed on ice all the time (Time 0 ). At the end of endocytosis, cells were cooled to $4{ }^{\circ} \mathrm{C}$ quickly by rinsing twice with cold HBSS; $\mathrm{F} c \alpha \mathrm{R}$ remaining on cell surface after endocytosis were stained with FITC-conjugated goat anti-mouse IgG by incubating in $1 \%$ $\mathrm{BSA} / \mathrm{PBS} / \mathrm{AZ}$ at $4{ }^{\circ} \mathrm{C}$ for $45 \mathrm{~min}$. Then, cells were washed with cold $1 \% \mathrm{BSA} / \mathrm{PBS} / \mathrm{AZ}$ thrice and analyzed by flow cytometry
(CHO cells were detached by trypsin digestion before analysis). The percentage of FcaR endocytosis was calculated as: (MIP8a on cell surface at $4{ }^{\circ} \mathrm{C}$ (Time 0 )-MIP8a on cell surface after $37{ }^{\circ} \mathrm{C}$ incubation)/ MIP8a on cell surface at $4{ }^{\circ} \mathrm{C}$ (Time 0$) \times 100 \%$.

\section{Immunofluoresence and confocal microscopy}

$\mathrm{CHO}$ cells stably expressing Fc $\alpha \mathrm{R}$ were allowed to grow on coverslip as described above. $24 \mathrm{~h}$ later, cells were transfected with EGFP-tagged Eps15-EH29, DIII, D3 $\Delta 2$, HA-tagged dynamin WT, dynamin K44A or EGFP-tagged Rab GTPases. In shRNA experiment, Hela cells were cotransfected with pcDNA3.1-Fc $\alpha$ R and pSUPER-shRNA CHC at the ratio of $1: 1$. At $48 \mathrm{~h}$ posttransfection, cells were cooled to $4{ }^{\circ} \mathrm{C}$ and incubated with FITC or TRITClabeled MIP8a-F $\left(a b^{\prime}\right)_{2}$ in medium at $4{ }^{\circ} \mathrm{C}$ for $60 \mathrm{~min}$, then washed with cold HBSS and transferred quickly into pre-warmed $\left(37^{\circ} \mathrm{C}\right)$ medium, followed by incubation at $37^{\circ} \mathrm{C}$ for various times. At the end of endocytosis, cells were placed on ice and fixed with $4 \%$ paraformaldehyde. To detect HA and clathrin, cells were permeabilized by $0.2 \%$ Triton $\mathrm{X}-100$ for $10 \mathrm{~min}$ at room temperature, then blocked with $5 \%$ horse serum for $30 \mathrm{~min}$. Cells were incubated with rabbit anti-clathrin heavy chain polyclonal antibody or goat anti-HA polyclonal antibody in $1 \% \mathrm{BSA} / \mathrm{PBS}$ for $60 \mathrm{~min}$ at room temperature, then washed with $1 \% \mathrm{BSA} / \mathrm{PBS}$, followed by incubation with FITC-conjugated goat anti-rabbit IgG or rabbit antigoat $\mathrm{IgG}$ for another $60 \mathrm{~min}$ at room temperature. Then, cells were washed and nuclei were stained with Hoechst 33258 (Merck \& Co, USA). The colocalization of endocytosed MIP8a-F( $\left.\mathrm{ab}^{\prime}\right)_{2}$ and Rabs GTPases during various time points was determined by a confocal laser-scanning microscope system (Leica TCS SP2 SE, Germany) with $100 \times 1.44$ numerical aperture oil immersion lens. The effects of Eps15, dynamin and Rabs GTPases on FcaR endocytosis were also examined by confocal microscopy. Each image was a single confocal slice. For quantitative analysis, the fluorescence intensity of endocytosed TRITC-conjugated MIP8a-F $\left(\mathrm{ab}^{\prime}\right)_{2}$ within cells were quantified by Image J software (NIH, USA). In each experiment, 100 untransfected cells and 100 transfected cells were quantified for their endocytosis of TRITC-conjugated MIP8a-F $\left(\mathrm{ab}^{\prime}\right)_{2}$, and the results were expressed as mean \pm SEM.

\section{Transferrin endocytosis assay}

First, cells were serum starved by incubation in medium without serum for $1 \mathrm{~h}$ at $37^{\circ} \mathrm{C}$. Then, cells were incubated with $50 \mu \mathrm{g} /$ $\mathrm{ml}$ Texas Red-Tfn in medium without serum at $37{ }^{\circ} \mathrm{C}$ for various times. At the end of endocytosis, cells were cooled to $4{ }^{\circ} \mathrm{C}$ quickly and washed with cold HBSS. Then, cells were fixed, stained with Hoechst 33258, mounted and analyzed as described above.

\section{$R N A$ interference}

Target sequence of shRNA duplexes against CHC (GTA ATC CAA TTC GAA GAC C) was obtained from a published study [56]. For the expression of shRNA, oligonucleotides containing target sequence to $\mathrm{CHC}$ were synthesized and duplex oligo DNA was inserted into the pSUPER vector (Oligoengine, USA). This plasmid DNA was cotransfected with pcDNA3.1-Fc $\alpha$ R in Hela cells. At $72 \mathrm{~h}$ posttransfection, the efficiency of $\mathrm{CHC}$ depletion and its influence on $\mathrm{Fc} \alpha \mathrm{R}$ endocytosis were examined by confocal microscopy. To knockdown CHC in U937 cells, a lentiviral shRNA vector pLVTHM [57] was used. pLVTHM expresses GFP and shRNA simultaneously, therefore infected cells can be easily detected by 
fluorescent microscopy and also can be sorted by FACS. The same target sequence of CHC was cloned in pLVTHM. pLVTHM or pLVTHM-shRNA CHC was cotransfected with packaging vector psPAX2 and envelope vector pMD2.G into 293T cells. Then U937 cells were infected with supernatants from packing cells supplemented with $8 \mu \mathrm{g} / \mathrm{ml}$ Polybrene. After 3 infections, cells with high GFP expression level were sorted by FACS. The expression of CHC in GFP-positive U937 cells was determined by western blot and fluorescent staining.

\section{Western blot}

To validate Fc $\alpha \mathrm{R}$ stably expressed in $\mathrm{CHO}$ cells, Fc $\alpha \mathrm{R}$ from $5 \times 10^{6}$ cells was purified with anti-Fc $\alpha \mathrm{R}$ mAb-coupled beads as described previously [50], separated by $10 \%$ SDS-PAGE and transferred onto a nitrocellulose membrane. After blocking with 5\% defatted milk for $1 \mathrm{~h}$ at room temperature, membranes were incubated with rabbit anti-Fc $\alpha$ R polyclonal antibody (1: 500) overnight at $4{ }^{\circ} \mathrm{C}$. Then, membranes were washed with PBS containing $0.05 \%$ Tween-20 and incubated with HRP-conjugated goat anti-rabbit $\operatorname{IgG}(1: 3000)$ at room temperature for $1 \mathrm{~h}$. Then membranes were visualized by SuperSignal West Pico chemiluminescent substrate (Pierce, USA). In RNA interference experiment, depletion of CHC was also examined by western blot. Briefly, $72 \mathrm{~h}$ posttransfection, equal number of pSUPER or pSUPER-shRNA CHC-transfected cells was lysed with loading buffer and separated by 5\%-18\% SDS-PAGE, then proteins were transferred onto a nitrocellulose membrane. Mouse monoclonal anti-CHC antibody (TD-1) was used to detect CHC (1:200).

\section{Acknowledgments}

This research was supported by grants from the National Natural Science Foundation of China (30170878 and 30571693). The authors thank Jane Brill for her language review of this manuscript. We greatly appreciate the gift of EGFP-tagged Rab7, Rab9 and Rab11 from Dr Richard E Pagano, EGFP-tagged Rab5 from Dr Feng Du, EGFP-tagged Eps 15 from Dr Alexandre Benmerah and HA-tagged dynamin from Dr Sandra Schmid. We also thank Dr Didier Trono for providing the lentiviral shRNA vector pLVTHM.

\section{References}

1 Conley ME, Delacroix DL. Intravascular and mucosal immunoglobulin A: two separate but related systems of immune defense? Ann Intern Med 1987; 106:892-899.

2 Mestecky J, Russell MW. Mucosal immunoglobulins and their contribution to defence mechanisms: an overview. Biochem Soc Trans 1997; 25:457-462.

3 Hellwig SM, van Spriel AB, Schellekens JF, et al. Immunoglobulin A-mediated protection against Bordetella pertussis infection. Infect Immun 2001; 69:4846-4850.

4 van Egmond $M$, van Garderen E, van Spriel AB, Damen CA et al. FcaRI-positive liver Kupffer cells: reappraisal of the function of immunoglobulin A in immunity. Nat Med 2000; 6:680-685.

5 Monteiro RC, van de Winkel JG. IgA Fc receptors. Annu Rev Immunol 2003; 21:177-204.

6 Ravetch JV, Bolland S. IgG Fc receptors. Annu Rev Immunol
2001; 19:275-290.

7 Stewart WW, Mazengera RL, Shen L, et al. Unaggregated serum IgA binds to neutrophil Fc $\alpha \mathrm{R}$ at physiological concentrations and is endocytosed but cross-linking is necessary to elicit a respiratory burst. J Leukoc Biol 1994; 56:481-487.

8 Morton HC, van den Herik-Oudijk IE, Vossebeld P, et al. Functional association between the human myeloid immunoglobulin A Fc receptor (CD89) and FcR $\gamma$ chain. J Biol Chem 1995; 270:29781-29787.

9 Lang ML, Shen L, Wade WF. $\gamma$ chain dependent recruitment of tyrosine kinases to membrane rafts by the human IgA receptor FcaR. J Immunol 1999; 163:5391-5398.

10 Lang ML, Chen YW, Shen L, et al. IgA Fc receptor $(\mathrm{Fc} \alpha \mathrm{R})$ cross-linking recruits tyrosine kinases, phosphoinositide kinases and serine/threonine kinases to glycolipid rafts. Biochem J 2002; 364:517-525.

11 Amigorena S, Salamero J, Davoust J, et al. Tyrosine-containing motif that transduces cell activation signals also determines internalization and antigen presentation via type III receptors for IgG. Nature 1992; 358:337-341.

12 Launay P, Patry C, Lehuen A, et al. Alternative endocytic pathway for immunoglobulin A Fc receptors (CD89) depends on the lack of FcR $\gamma$ association and protects against degradation of bound ligand. J Biol Chem 1999; 274:7216-7225.

13 Shen L, van Egmond M, Siemasko K, et al. Presentation of ovalbumin internalized via the immunoglobulin-A Fc receptor is enhanced through Fc receptor $\gamma$ chain signaling. Blood 2001; 97:205-213.

14 Conner SD, Schmid SL. Regulated portals of entry into the cell. Nature 2003; 422:37-44.

15 Parton RG, Richards AA. Lipid rafts and caveolae as portals for endocytosis: new insights and common mechanisms. Traffic 2003 ; 4:724-738.

16 Kirkham M, Parton RG. Clathrin-independent endocytosis: new insights into caveolae and non-caveolar lipid raft carriers. Biochim Biophy Acta 2005; 1746:349-363.

17 Hill E, van Der Kaay J, Downes CP, et al. The role of dynamin and its binding partners in coated pit invagination and scission. J Cell Biol 2001; 152:309-323.

18 Sever S, Damke H, Schmid SL. Dynamin: GTP controls the formation of constricted coated pits, the rate limiting step in clathrin-mediated endocytosis. J Cell Biol 2000; 150:11371148 .

19 Damke H, Baba T, Warnock DE, et al. Induction of mutant dynamin specifically blocks endocytic coated vesicle formation. J Cell Biol 1994; 127:915-934.

20 Mayor S, Pagano RE. Pathways of clathrin-independent endocytosis. Nat Rev Mol Cell Biol 2007; 8:603-612.

21 Fattakhova G, Masilamani M, Borrego F, et al. The highaffinity immunoglobulin-E receptor $\left(\mathrm{Fc}_{\varepsilon} \mathrm{RI}\right)$ is endocytosed by an AP-2/clathrin-independent, dynamin-dependent mechanism. Traffic 2006; 7:673-685.

22 Somsel Rodman J, Wandinger-Ness A. Rab GTPases coordinate endocytosis. J Cell Sci 2000; 113:183-192.

23 Mohrmann K, van der Sluijs P. Regulation of membrane transport through the endocytic pathway by rab GTPases. Mol Membr Biol 1999; 16:81-87.

24 Nielsen E, Severin F, Backer JM, et al. Rab5 regulates motility of early endosomes on microtubules. Nat Cell Biol 1999; 
1:376-382.

25 Simonsen A, Lippé R, Christoforidis S, et al. EEA1 links $\mathrm{PI}(3) \mathrm{K}$ function to Rab5 regulation of endosome fusion. $\mathrm{Na}$ ture 1998; 394:494-498.

26 Chen W, Feng Y, Chen D, et al. Rab11 is required for transGolgi network-toplasma membrane transport and a preferential target for GDP dissociation inhibitor. Mol Biol Cell 1998; 9:3241-3257.

27 Hamelin E, Thériault C, Laroche G, et al. The intracellular trafficking of the $\mathrm{G}$ protein-coupled receptor TPbeta depends on a direct interaction with Rab11. J Biol Chem 2005; 280:36195-36205.

28 Moore RH, Millman EE, Alpizar-Foster E, et al. Rab11 regulates the recycling and lysosome targeting of beta ${ }_{2}$-adrenergic receptors. J Cell Sci 2004; 117:3107-3117.

29 Feng Y, Press B, Wandinger-Ness A. Rab 7: an important regulator of late endocytic membrane traffic. J Cell Biol 1995; 131:1435-1452.

30 Bucci C, Thomsen P, Nicoziani P, et al. Rab7: a key to lysosome biogenesis. Mol Biol Cell 2000; 11:467-480.

31 Lombardi D, Soldati T, Riederer MA, et al. Rab9 functions in transport between late endosomes and the trans-Golgi network. EMBO J 1993; 12:677-682.

32 Barbero P, Bittova L, Pfeffer SR. Visualization of Rab9mediated vesicle transport from endosomes to the trans-Golgi in living cells. J Cell Biol 2002; 156:511-518.

33 Seachrist JL, Ferguson SS. Regulation of G protein-coupled receptor endocytosis and trafficking by Rab GTPases. Life Sci 2003; 74:225-235.

34 Heuser JE, Anderson RG. Hypertonic media inhibit receptormediated endocytosis by blocking clathrin-coated pit formation. J Cell Biol 1989; 108:389-400.

35 Phonphok Y, Rosenthal KS. Stabilization of clathrin coated vesicles by amantadine, tromantadine and other hydrophobic amines. FEBS Lett 1991; 281:188-190.

36 Altankov G, Grinnell F. Depletion of intracellular potassium disrupts coated pits and reversibly inhibits cell polarization during fibroblast spreading. J Cell Biol 1993; 120:1449-1459.

37 Ros-Baro A, Lopez-Iglesias C, Peiro S, et al. Lipid rafts are required for GLUT4 internalization in adipose cells. Proc Natl Acad Sci USA 2001; 98:12050-12055.

38 Orlandi PA, Fishman PH. Filipin-dependent inhibition of cholera toxin: evidence for toxin internalization and activation through caveolae-like domains. J Cell Biol 1998; 141:905915.

39 Singh RD, Puri V, Valiyaveettil JT, et al. Selective caveolin1-dependent endocytosis of glycosphingolipids. Mol Biol Cell 2003; 14:3254-3265.

40 Minshall RD, Tiruppathi C, Vogel SM, et al. Endothelial cellsurface gp60 activates vesicle formation and trafficking via G(i)-coupled Src kinase signaling pathway. J Cell Biol 2000; 150:1057-1070.

41 Sharma DK, Brown JC, Choudhury A, et al. Selective stimulation of caveolar endocytosis by glycosphingolipids and cholesterol. Mol Biol Cell 2004; 15:3114-3122.

42 Damm EM, Pelkmans L, Kartenbeck J, et al. Clathrin- and caveolin-1-independent endocytosis: entry of simian virus 40 into cells devoid of caveolae. J Cell Biol 2005; 168:477-488.

43 Benmerah A, Bayrou M, Cerf-Bensussan N, et al. Inhibition of clathrin-coated pit assembly by an Eps 15 mutant. J Cell Sci 1999; 112:1303-1311.

44 Bonifacino JS, Traub LM. Signals for sorting of transmembrane proteins to endosomes and lysosomes. Annu Rev Biochem 2003; 72:395-447.

45 Davis W, Harrison PT, Hutchinson MJ, et al. Two distinct regions of $\mathrm{Fc} \gamma \mathrm{RI}$ initiate separate signalling pathways involved in endocytosis and Phagocytosis. EMBO J 1995; 14:432-441.

46 Mero P, Zhang CY, Huang ZY, et al. Phosphorylation-independent ubiquitylation and endocytosis of Fc $\gamma$ RIIA. $J$ Biol Chem 2006; 281:33242-33249.

47 Dinneen JL, Ceresa BP. Expression of dominant negative rab5 in HeLa cells regulates endocytic trafficking distal from the plasma membrane. Exp Cell Res 2004; 294(2):509-522.

48 Seachrist JL, Laporte SA, Dale LB, et al. Rab5 association with the angiotensin II type 1A receptor promotes Rab5 GTP binding and vesicular fusion. J Biol Chem 2002; 277:679-685.

49 Leser GP, Ector KJ, Ng DT, et al. The signal for clathrinmediated endocytosis of the paramyxovirus SV5 HN protein resides at the transmembrane domain-ectodomain boundary region. Virology 1999; 262:79-92.

50 Guo Y, Smith K, Lee J, et al. Identification of methionine-rich clusters that regulate copper-stimulated endocytosis of the human Ctr1 copper transporter. J Biol Chem 2004; 279:1742817433.

51 Patry C, Sibille Y, Lehuen A, et al. Identification of Fc $\alpha$ receptor (CD89) isoforms generated by alternative splicing that are differentially expressed between blood monocytes and alveolar macrophages. J Immunol 1996; 156:4442-4448.

52 Zhang W, Bi B, Oldroyd RG, et al. Neutrophil lactoferrin release induced by IgA immune complexes differed from that induced cross-linking of fcalpha receptors $(\mathrm{Fc} \alpha \mathrm{R})$ with a monoclonal antibody, MIP8a. Clin Exp Immunol 2000; 121:106-111.

53 Yin N, Peng M, Xing Y, et al. Intracellular pools of Fc $\alpha$ R (CD89) in human neutrophils are localized in tertiary granules and secretory vesicles, and two $\mathrm{Fc} \alpha \mathrm{R}$ isoforms are found in tertiary granules. J Leukoc Biol 2007; 82:551-558.

54 Brüggemann M, Williams GT, Bindon CI, et al. Comparison of the effector functions of human immunoglobulins using a matched set of chimeric antibodies. J Exp Med 1987;166:1351-1361.

55 Choudhury A, Dominguez M, Puri V, et al. Rab proteins mediate Golgi transport of caveola-internalized glycosphingolipids and correct lipid trafficking in Niemann-Pick C cells. $J$ Clin Invest 2002; 109:1541-1550.

56 Enari M, Ohmori K, Kitabayashi I, et al. Requirement of clathrin heavy chain for p53-mediated transcription. Genes Dev 2006; 20:1087-1099.

57 Wiznerowicz M, Trono D. Conditional suppression of cellular genes: lentivirus vector-mediated drug-inducible RNA interference. J Virol 2003; 77:8957-8961.

(Supplementary information is linked to the online version of the paper on the Cell Research website.) 